\title{
Higher spin supersymmetry at the cosmological collider: sculpting SUSY rilles in the CMB
}

\author{
Stephon Alexander, ${ }^{a, b}$ S. James Gates Jr., ${ }^{a, b}$ Leah Jenks, ${ }^{a}$ K. Koutrolikos ${ }^{a}$ \\ and Evan McDonough ${ }^{a}$ \\ ${ }^{a}$ Department of Physics, Brown University, \\ Box 1843, 182 Hope Street, Barus 6 Holley 545, Providence, RI 02912, U.S.A. \\ ${ }^{b}$ Brown Theoretical Physics Center, \\ Providence, RI 02912, U.S.A. \\ E-mail: stephon_alexander@brown.edu, sylvester_gates@brown.edu, \\ leah_jenks@brown.edu, konstantinos_koutrolikos@brown.edu, \\ evan_mcdonough@brown.edu
}

ABSTRACT: We study the imprint of higher spin supermultiplets on cosmological correlators, namely the non-Gaussianity of the cosmic microwave background. Supersymmetry is used as a guide to introduce the contribution of fermionic higher spin particles, which have been neglected thus far in the literature. This necessarily introduces more than just a single additional fermionic superpartner, since the spectrum of massive, higher spin supermultiplets includes two propagating higher spin bosons and two propagating higher spin fermions, which all contribute to the three point function. As an example we consider the half-integer superspin $\mathrm{Y}=s+1 / 2$ supermultiplet, which includes particles of spin values $j=s+1, j=s+1 / 2, j=s+1 / 2$ and $j=s$. We compute the curvature perturbation 3-point function for higher spin particle exchange and find that the known $P_{s}(\cos \theta)$ angular dependence is accompanied by superpartner contributions that scale as $P_{s+1}(\cos \theta)$ and $\sum_{m} P_{s}^{m}(\cos \theta)$, with $P_{s}$ and $P_{s}^{m}$ defined as the Legendre and Associated Legendre polynomials respectively. We also compute the tensor-scalar-scalar 3-point function, and find a complicated angular dependence as an integral over products of Legendre and associated Legendre polynomials.

KEYwords: Cosmology of Theories beyond the SM, Higher Spin Symmetry, Supersymmetric Effective Theories

ARXIV EPRINT: 1907.05829 


\section{Contents}

1 Introduction 1

2 Setup in effective field theory 3

$\begin{array}{lll}3 & \text { Supersymmetric higher spins } & 7\end{array}$

4 Higher spin supersymmetry at the cosmological collider 10

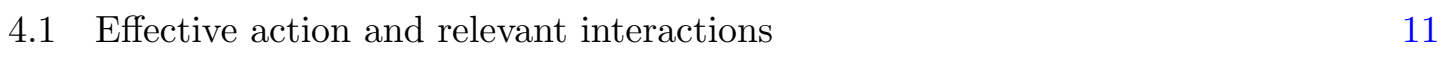

$\begin{array}{lll}4.2 & \text { Higher spin fields in de Sitter space } & 12\end{array}$

$\begin{array}{lll}4.3 & \text { Non-Gaussianity from higher spin particle exchange } & 15\end{array}$

$\begin{array}{ll}4.4 \text { The predictions of higher spin supersymmetry } & 19\end{array}$

$\begin{array}{lll}5 & \text { Discussion } & 20\end{array}$

$\begin{array}{lll}\text { A Spin- } s \text { polarization vectors } & 22\end{array}$

B Details of $\langle\gamma \zeta \zeta\rangle$ calculation $\quad 23$

\section{Introduction}

Inflationary cosmology provides the initial conditions of standard cosmology, and a mechanism to explain the origin of the large scale structure of the universe. These initial conditions are manifest in the statistical properties of anisotropies in the cosmic microwave background $(\mathrm{CMB})$ radiation, which in addition to being measured to an incredible precision [1], are well described by linearized cosmological perturbation theory. This latter fact means the statistical properties of the $\mathrm{CMB}$ are calculable, and combined with the conservation of the primordial curvature perturbation on super-horizon scales [2-8], makes possible deductions as to the precise particle content of the very early universe.

In particular, while single-field slow-roll inflation predicts adiabatic, Gaussian, nearlyscale invariant perturbations, interactions of the primordial curvature perturbation with new fields can generate deviations from Gaussianity, as encoded at lowest-order by the 3-point function $\langle\zeta \zeta \zeta\rangle$. Remarkably, this is sensitive even to fields that are heavier than the Hubble scale during inflation [9-11], and thus probes particles at energy scales far above those accessible by terrestrial colliders. The study of $\langle\zeta \zeta \zeta\rangle$ as a particle detector has been termed 'Cosmological Collider Physics' [12] (see also [13]), and gained significant momentum due to the realization that interactions with higher spin bosons, namely the exchange of a massive spin- $s$ boson, impart a characteristic angular dependence on the non-Gaussianity, $\left\langle\zeta\left(k_{1}\right) \zeta\left(k_{2}\right) \zeta\left(k_{3}\right)\right\rangle \propto P_{s}\left(\hat{k}_{1} \cdot \hat{k}_{3}\right)+k_{2} \leftrightarrow k_{3}$, with $P_{s}(\cos \theta)$ the degree- $s$ Legendre polynomial. 
The study of higher spins has a long history dating all the way back to the founding of relativistic field theory. ${ }^{1}$ Since then, higher spins have gained fame and attention in large part due to their role in string theory, ${ }^{2}$ as well as their use in exploring the holographic principle. ${ }^{3}$ Additionally, there is the old conjecture [17-20] that physics beyond Planckian energy scales will have higher symmetries emerging. From this point of view the study of higher spins can be understood as an attempt to classify and realize the various possibilities for these emerging symmetries. The study of manifestly supersymmetric higher spins is a natural extension of the above program, both because supersymmetry is a property of the underlying theory (as in the example of (super)string theory) and, more generally, because it is compatible with the relevant structures (like the symmetries of S-matrix [21]).

For these reasons we are interested in using irreducible representations of the supersymmetric extension of appropriate spacetime symmetry groups which involve higher spin particles. These irreps are classified and labeled by the eigenvalues of the Casimir Operators. In $4 D$ these are the mass $(m)$ and the superspin $(\mathrm{Y})$ which takes either integer $(\mathrm{Y}=s)$ or half integer values $(\mathrm{Y}=s+1 / 2) .{ }^{4}$ These representations include multiple representations of the non-supersymmetric spacetime symmetry group: for the massless case $(m=0)$ a supermultiplet with superspin $\mathrm{Y}$ includes massless particles with spins $j=\mathrm{Y}+1 / 2$ and $j=\mathrm{Y}$, whereas for the massive case $(m \neq 0)$ a supermultiplet with superspin $\mathrm{Y}$ includes massive particles with spins $j=\mathrm{Y}+1 / 2, j=\mathrm{Y}, j=\mathrm{Y}$ and $j=\mathrm{Y}-1 / 2$. This implies that supersymmetrizing the cosmological collider does not simply require adding a single higher spin fermion, but rather additional fields as well.

As a step towards combining this with inflationary cosmology, we consider an inflationary sector minimally coupled to a higher spin sector. The inflationary vacuum energy $H^{2}$ breaks supersymmetry, generating masses for the inflationary fermionic superpartners, while the higher spin sector, behaving as 'spectator fields' which do not contribute to $H^{2}$ and hence do not contribute to supersymmetry breaking, retain their on-shell supersymmetry. Given the candidate bosonic interactions proposed in the literature [12, 13], the remnant on-shell supersymmetry of the higher spin sector uniquely determines the interactions of the higher spin fermions with the primordial curvature perturbation. From this one can compute the statistical properties of anisotropies in the CMB, and in this way, use the CMB as a detector for higher spin supersymmetry at the early universe's collider.

Each higher spin particle, as enumerated by the corresponding irreducible representation, induces a contribution to the 3-point function $\langle\zeta \zeta \zeta\rangle$, i.e. a signal at the cosmological collider, and in this work we explicitly calculate the non-Gaussianity due to these contributions. Our primary result is the prediction of higher spin supersymmetry for the

\footnotetext{
${ }^{1}$ First paper by Majorana in 1932 [14] followed by Dirac, Pauli, Fierz, Wigner and others.

${ }^{2}$ For example, the UV softness of perturbative string scattering amplitudes originates from the freedom to exchange higher spin particles. More recently, higher spin fields have played a role in constraining the self-consistency of inflation in string theory $[15,16]$.

${ }^{3}$ All available, consistent, fully interacting higher spin theories (such as Vasiliev's or CS in 3D) require an AdS background and a spin two state. Both of these requirements are ingredients of AdS/CFT correspondence.

${ }^{4}$ For the purpose of this discussion we will ignore infinite sized representations that go under the name of continuous (super)spin representations [22].
} 
angular dependence of the non-Gaussianity: we find that the $P_{s}$ contributions to the nonGaussianity come in a characteristic pattern. Namely, every $P_{s}$ contribution to the nonGaussianity is accompanied by a $P_{s+1}$ contribution and a tower of associated Legendre polynomials $P_{s}^{m}$. The magnitudes, while Boltzmann suppressed, are related by supersymmetric considerations.

This paper is organized in the following way. In section 2 we build the effective field theory that will be the framework for our calculations. Section 3, gives a very elementary review of massive, higher superspin supermultiplets focusing on the spectrum of propagating spin particles they include. Furthermore it demonstrates how to construct a supersymmetric extension of the previously studied class of effective field theories. In section 4, using these types of fermionic higher spin terms we consider an effective interacting Lagrangian up to first order in the higher spin fields. The effective Lagrangian is then used to calculate the contribution of higher spin fermions to the three point function $\langle\zeta \zeta \zeta\rangle$. The last section 5 , gives a summary of our results and a short discussion for future directions, including the tensor-scalar-scalar 3-point function $\langle\gamma \zeta \zeta\rangle$, which we compute for higher spin fermion exchange.

\section{Setup in effective field theory}

In this work we consider a tripartite marriage of $4 D, \mathcal{N}=1$ supersymmetric higher spins [23-37] with the effective field theory (EFT) of inflation [38] and de Sitter supergravity [39-41]. We construct an effective field theory of a supersymmetric theory of higher spins in a quasi-de Sitter spacetime with spontaneously broken supersymmetry and spontaneously broken time-translation invariance. The goal of this construction is to minimally couple the higher spin sector and the inflationary sector, in such a way that the on-shell supersymmetry of the higher spin fields is maintained, despite supersymmetry being broken by the inflationary vacuum energy. The on-shell supersymmetry of the higher spin sector can then be used in conjunction with the effective field theory of inflation to dictate the couplings of higher spin fermions and bosons to primordial perturbations. This is distinct from the supersymmetric EFT of inflation [42] in that we do not focus on the gravity multiplet, but instead on the higher spin supermultiplets.

Our approach allows us to make progress despite not having a full theory of interacting higher spin de Sitter supergravity. While the setup may seem contrived, it bears some similarity with the interplay of supersymmetry and anomaly cancellation in string theory.

To appreciate this, one may recall that the interactions between effective field theory, supersymmetry and anomaly cancellation are not as direct as one might imagine. In some cases a complete superspace formulation or component-level supersymmetrization is known such as in the example of the $4 D, \mathcal{N}=1 \mathrm{WZNW-QCD}$ action [43-45]. In the development of Heterotic theory, anomaly cancellation required the addition of a new term in the action a la the famous Green-Schwartz mechanism [46], and this term required yet more terms (and years of calculation) in order to restore supersymmetry to the action [47]. Similarly, in Type II/M theories the gravitational anomaly on D/M-branes induced by loops of chiral fermions is canceled via anomaly inflow by a higher-derivative correction to the bulk action [48-50], 
and despite the anomaly not playing any direct role in supersymmetry, as for Green-Schwarz the supersymmetrization of the anomaly-canceling terms requires yet more terms be added, the calculation of which requires a herculean level of technical skill and detail [51]. The same issue applies to the $\mathrm{SL}(2, \mathbb{Z})$ symmetry of type IIB: restoring the invariance naively broken by the corrections requires the careful consideration of D-instantons [52] (and again these new terms must be supersymmetrized).

In each of these cases, a seemingly complete theory is found to be anomalous, and cancellation of the anomalies requires new terms. The new terms should respect the symmetries of the action, and generically additional terms must be found to accomplish this task. However, much can be learned even without a complete knowledge of all terms in the theory. For example, in the interim period between [48-50] and [51], the AdS/CFT correspondence was discovered [53]. Another example of this is of course the field of String Cosmology $[54,55]$, which makes no recourse to the precise manner in which $\operatorname{SL}(2, \mathbb{Z})$ symmetry is maintained. With all this in mind, we construct an effective theory along the lines discussed above.

This approach can be illustrated with simple examples involving chiral superfields in $\mathcal{N}=1$ supersymmetry. The first non-trivial step is the 'sequestering' of supersymmetry breaking to the inflationary sector, analogous to the Randall-Sundrum scenario [56]. This can be done in a number of ways; one simple example is to take guidance from $[57,58]$ and allow for a non-minimal Kahler potential, as in

$$
W=M X, \quad K=X \bar{X} e^{Y \bar{Y}}+Y \bar{Y} .
$$

This exhibits a vacuum at $X=\bar{X}=Y=\bar{Y}=0$, wherein supersymmetry is broken by $X$, $D_{X} W=M$. The scalar potential evaluated for $X=\bar{X}=0$ is given by a constant $V=M^{2}$, leaving the scalar component of $Y$ massless: $m_{Y}^{2} \equiv \partial_{Y \bar{Y}} V=0 .{ }^{5}$ Similarly, the fermion component of $Y$ remains massless since $D_{Y} W=0$. Thus the breaking of supersymmetry is not communicated to the on-shell mass spectra of $Y$, and the $Y$ superfield retains on-shell $\mathcal{N}=1$ supersymmetry.

The utility of this approach is the enumeration of interactions and the tree-level couplings, since despite the sequestering of supersymmetry breaking, there are interactions between $X$ and $Y$, which communicate the SUSY breaking at loop-level. Expanding $X \rightarrow \delta x$ and $Y \rightarrow \delta y, \delta x, \delta y \in \mathbb{R}$, one finds the interactions between scalar components,

$$
\mathcal{L}_{\text {int }}=\delta y^{2}(\partial \delta x)^{2}+M^{2} \delta y^{2} \delta x^{4}+\ldots
$$

where the ... are higher order terms. Similarly, there are interactions between the fermionic components of $X$ and the fermionic components of $Y$, and these two sets of interactions will communicate the SUSY breaking to $Y$. The structure of these interactions is governed by the underlying supersymmetry, which is spontaneously broken by $X$, and this structure dictates the effect that $\delta y$ interactions can have on $\delta x$ correlators.

\footnotetext{
${ }^{5}$ For this simple example, also the scalar component of $X$ is massless, but it can be made massive via an addition to the Kahler potential $\delta K=(X \bar{X})^{2} / \Lambda$. The fermionic component of $X$ has mass set by $M$.
} 
In our setup, the higher spin sector is analogous to $Y$ while the inflationary sector is analogous to $X$. It is the above sense in which the HS sector in our setup has on-shell supersymmetry. This can be used to enumerate the interactions and estimate the amplitude of correlation functions. However, this is not the full story: the next puzzle piece is the embedding of supersymmetry and supergravity into cosmological spacetimes.

This can be done via the framework of de Sitter supergravity [39-41]. This theory describes the spontaneous breaking of supersymmetry with no field content other then the gravity multiplet and the goldstino of supersymmetry breaking. The latter can be expressed as a chiral superfield, $S$, satisfying a constraint equation,

$$
S^{2}=0
$$

This constraint removes the scalar degree of freedom from $S$, leaving the fermionic component as the only propagating degree of freedom. The most general superpotential is given by,

$$
W=W_{0}+M S
$$

since any additional terms involving $S$ vanish by the nilpotency constraint. Supersymmetry is broken by $D_{S} W=M$, and the resulting scalar potential, for a minimal Kahler potential $K=S \bar{S}$, is a cosmological constant given by

$$
\Lambda \equiv V=M^{2}-3 W_{0}^{2}
$$

which is positive for $M>\sqrt{3} W_{0}$, giving a de Sitter spacetime.

Any additional matter sectors in de Sitter supergravity can easily be sequestered from the breaking of supersymmetry. For example, endowing $S$ with a non-trivial Kahler geometry $[57,58]$ and taking $W_{0}=0$,

$$
W=M S, \quad K=e^{T \bar{T}} S \bar{S}+T \bar{T}, \quad S^{2}=0,
$$

supersymmetry-breaking is purely in the $S$-direction provided that $D_{T} W=0$ in vacuum, which is guaranteed to be the case since $D_{T} W \propto S=0$, leaving the fermionic component of $T$ massless. Meanwhile, SUSY is broken by $S, D_{S} W=M$, and the potential is a constant vacuum energy $V=\Lambda=M$, leaving the scalar components of $T$ massless. Thus again, $T$ retains on-shell supersymmetry.

To connect this with observational cosmology, and anisotropies in the cosmic microwave background radiation, it is necessary to consider fluctuations. Inflation models can be constructed in de Sitter supergravity along the lines of [57-60]. Consider a superfield $\Phi$ with the real part of the scalar component of $\Phi$ identified as the inflaton $\varphi$. The fluctuations of $\varphi$ in spatially flat gauge are related to the curvature perturbation on uniform density hypersurfaces $\zeta$ in uniform field gauge via [61]

$$
\zeta=\frac{H}{\dot{\varphi}} \delta \varphi=\frac{1}{\sqrt{2 \epsilon} m_{p l}} \delta \varphi,
$$


where $\epsilon \equiv-\dot{H} / H^{2}$ is the inflationary slow-roll parameter. This defines the primordial power spectrum, in dimensionless form,

$$
\Delta_{\zeta}^{2} \equiv \frac{k^{3}}{2 \pi^{2}}\left|\zeta_{k}\right|^{2}
$$

where $\zeta_{k}$ is a Fourier mode of the field $\zeta(x, t)$.

The curvature perturbation $\zeta$ can in turn be related to the Goldstone boson of spontaneously broken time-translation invariance, using the machinery of the effective field theory of inflation [38]. This starts from the realization that the time-dependence of the inflaton $\varphi(t)$ breaks the time diffeomorphisms. The Goldstone boson can be included in the theory by a redefinition of the time-coordinate,

$$
t \rightarrow t-\pi(t, x)
$$

with $\pi(t, x)$ the Goldstone boson. This induces a field fluctuation,

$$
\varphi(t) \rightarrow \varphi(t)-\dot{\varphi} \pi(t, x)
$$

and thus corresponds to a curvature perturbation,

$$
\zeta=-H \pi \text {. }
$$

This also generates a fluctuation to the 00 component of the metric,

$$
\delta g^{00}=-2 \dot{\pi}
$$

The interactions of $\pi$, and hence $\zeta$, are dictated by the symmetry structure of the action, which is broken to invariance under spatial rotations. This allows $\delta g^{00}$ to appear explicitly in the action, while $\delta g^{i j}$ can only appear with all indices contracted.

Similarly, the interactions of $\pi$, and hence $\zeta$, with any additional fields are dictated by symmetry considerations. For fields with arbitrary spin, $\sigma_{\mu_{1} \ldots \mu_{s}}$, this leads to effective interactions of the form $[12,13]$

$$
\mathcal{L}_{\text {int }} \supset \frac{\lambda_{s}}{\Lambda^{s-3}} \partial_{i_{1}} \ldots \partial_{i_{s}} \zeta \sigma^{i_{1} \ldots i_{s}}+\frac{g_{s}}{\Lambda^{s-2}} \dot{\zeta} \partial_{i_{1}} \ldots \partial_{i_{s}} \zeta \sigma^{i_{1} \ldots i_{s}}
$$

where indices $i$ runs over spatial directions: $1,2,3$, and $\Lambda$ is a UV scale. These terms descend from higher-dimension operators built out of the metric and its derivatives, and have coupling constants that are a priori free parameters of the effective field theory. For example, in the spin- 2 case, these terms arise from a coupling of a spin- 2 field $\sigma$ to the extrinsic curvature, $\sqrt{-g} K^{\mu \nu} \sigma_{\mu \nu}$. The first term in (2.13) descends from $\delta K^{\mu \nu} \sigma_{\mu \nu}$ while the second term arises from including the metric perturbation $\delta g^{00} \delta K^{\mu \nu} \sigma_{\mu \nu}$ [13]. There can also be additional terms at higher order in $\pi$ and $\sigma$ and terms with different distribution of derivatives (up to total derivatives).

We now arrive back at the tripartite marriage: we wish to connect the higher spin interactions in a quasi-dS space to supersymmetry. To do this, one could simply operate along effective field theory lines, and introduce interactions consistent with unbroken spatial rotations. However, an interesting possibility is to consider what we can learn from higher spin supersymmetry, and use this as guidance in constructing our effective field theory describing the interactions of the higher spin fermions. Towards this end, we now develop the machinery of higher spin supersymmetry. 


\section{Supersymmetric higher spins}

The first Lagrangian description of supersymmetric, massless, higher spins in $4 D$ Minkowski space was done in $[62,63]$, using components with on-shell supersymmetry. A natural approach to the off-shell formulation is to use the superspace and superfield methods (see e.g. [64, 65]). A superfield description of free supersymmetric massless, higher spin theories was presented for the first time in [23-25] for both Minkowski and AdS spaces. This approach has been further explored in [26-29]. Later studies of free supersymmetric, massless higher spin supermultiplets include [30-33].

On the other hand, the Lagrangian description of $4 \mathrm{D}$ massive supersymmetric spins for arbitrary values of spin is only known in the component formulation with on-shell supersymmetry [34, 35], whereas the off-shell, superspace description has been developed up to superspin $Y=3 / 2$ supermultiplet [36, 37]. Nevertheless, independently of what the proper Lagrangian description is, we know that there are two types of such irreps $(i)$ the integer superspin $\mathrm{Y}=s$ supermultiplets and $(i i)$ the half integer superspin $\mathrm{Y}=s+1 / 2$ supermultiplets. Moreover we know that on-shell they describe two bosonic and two fermionic massive higher spin particles with spin values $j=\mathrm{Y}+1 / 2, j=\mathrm{Y}, j=\mathrm{Y}$ and $j=\mathrm{Y}-1 / 2$. The half integer superspin $Y=s+1 / 2$ supermultiplet, consisting of components

$$
\mathrm{Y}=s+1 / 2: \quad(s+1, s+1 / 2, s+1 / 2, s)
$$

and its on-shell, superspace description is given in terms of a real, bosonic superfield $H_{\alpha(s) \dot{\alpha}(s)},{ }^{6}$ with the following on-shell conditions:

$$
\mathrm{D}^{\alpha_{s}} H_{\alpha(s) \dot{\alpha}(s)}=0, \square H_{\alpha(s) \dot{\alpha}(s)}=m^{2} H_{\alpha(s) \dot{\alpha}(s)},
$$

where $D^{\alpha_{s}}$ is the superspace covariant derivative. Alternatively, the massive, integer $\mathrm{Y}=s$ superspin supermultiplet, comprised of components,

$$
\mathrm{Y}=s: \quad(s+1 / 2, s, s, s-1 / 2)
$$

has an on-shell superspace description in terms of a fermionic superfield $\Psi_{\alpha(s) \dot{\alpha}(s-1)}$ with the on-shell equations:

$$
\mathrm{D}^{\alpha_{s}} \Psi_{\alpha(s) \dot{\alpha}(s-1)}=0, \overline{\mathrm{D}}^{\dot{\alpha}_{s-1}} \Psi_{\alpha(s) \dot{\alpha}(s-1)}=0, i \partial_{\alpha_{s}}^{\dot{\alpha}_{s}} \bar{\Psi}_{\alpha(s-1) \dot{\alpha}(s)}+m \Psi_{\alpha(s) \dot{\alpha}(s-1)}=0 .
$$

We start with the assumption that the higher spin sector respects supersymmetry and therefore can be organized into higher spin supermultiplets. This is extremely useful because supersymmetry will guide us to the introduction of higher spin fermions which have been neglected so far. Once their contribution is better understood, one may choose to drop the assumption of supersymmetry and study these fermionic contributions independently.

\footnotetext{
${ }^{6}$ The notation $\alpha(s)$ signifies a string of $s$ undotted indices $\alpha_{1} \alpha_{2} \ldots \alpha_{s}$ which are symmetrized. This type of indices are the spinorial indices of a Weyl spinor of one chirality and take values 1 and 2 in $4 D$. Similarly for $\dot{\alpha}(s)$, where $\dot{\alpha}$ are the spinorial indices of the opposite chirality Weyl spinor and take also two values $\dot{1}$ and $\dot{2}$ in $4 D$.
} 
The strategy for finding the fermionic higher spin contributions is: (a) Start with the family of effective actions that lead to (2.13) after breaking the time translation invariance, and elevate them to superspace. This will automatically introduce all fermionic partners. (b) We project back down to a component description to reveal the interactions of the higher spin fermions. (c) Finally, we break supersymmetry appropriately in the inflaton sector.

The first step is to embed the bosonic, massive spin $s$ particle in a massive higher spin supermultiplet described by some higher spin superfield. As we have seen, there are two ways of doing that, we can either use the integer or the half-integer superspin supermultiplet, with components (3.3) and (3.1) respectively. For concreteness, we make the latter choice $(\mathrm{Y}=s+1 / 2)$ which means that our spin $s$ particle will be accompanied by one bosonic higher spin particle $j=s+1$ and two more fermionic higher spin particles $j=s+1 / 2$. In this choice the highest propagating spin is $s+1$. Similarly we embed the scalar curvature perturbation field in a scalar supermultiplet, which will of course introduce its fermionic superpartner, which we refer to as the inflatino. ${ }^{7}$ A simple choice to describe such a scalar supermultiplet is to use a chiral superfield $\Phi$.

Secondly, using superspace, we write quadratic and cubic interaction terms, between $H_{\alpha(s) \dot{\alpha}(s)}$ and $\Phi$ which are linear in the higher spin superfield. The family of such superspace effective Lagrangians takes the form:

$$
\mathcal{L}=H^{\alpha(s) \dot{\alpha}(s)} \mathcal{I}_{\alpha(s) \dot{\alpha}(s)}+H^{\alpha(s) \dot{\alpha}(s)} \mathcal{J}_{\alpha(s) \dot{\alpha}(s)}
$$

where $\mathcal{I}_{\alpha(s) \dot{\alpha}(s)}$ is linear in $\Phi$ and generates the quadratic interactions part, whereas $\mathcal{J}_{\alpha(s) \dot{\alpha}(s)}$ is quadratic in $\Phi$ and generates the cubic ${ }^{8}$ part of the interactions. The most general ansatzes for $\mathcal{I}_{\alpha(s) \dot{\alpha}(s)}$ and $\mathcal{J}_{\alpha(s) \dot{\alpha}(s)}$ are,

$$
\mathcal{I}_{\alpha(s) \dot{\alpha}(s)}=\partial^{(s)}\left(b \Phi+b^{*} \bar{\Phi}\right)
$$

and

$$
\mathcal{J}_{\alpha(s) \dot{\alpha}(s)}=\sum_{p=0}^{s}\left\{d_{p} \partial^{(p)} \Phi \partial^{(s-p)} \bar{\Phi}+f_{p} \partial^{(p)} \mathrm{D} \Phi \partial^{(s-p-1)} \overline{\mathrm{D}} \bar{\Phi}+g_{p} \partial^{(p)} \Phi \partial^{(s-p)} \Phi+g_{p}^{*} \partial^{(p)} \bar{\Phi} \partial^{(s-p)} \bar{\Phi}\right\} .
$$

Using this as a starting point, one can project the superspace Lagrangian to components and find the corresponding field theory (see [64,65] and detailed examples can be found in $[29,69])$. The result will include the entire spectrum of fields of the supersymmetric theory. In addition to the propagating spins, this includes the set of auxiliary

\footnotetext{
${ }^{7}$ In general the fermionic partner of the curvature perturbation field can be identified as a linear combination of the inflatino and other fermions in the theory.

${ }^{8}$ The reason why we are considering massive higher spin supermultiplets is a consequence of the Higuchi bound $[66,67]$ plus the possibility of higher order mass-like interaction terms for the higher spin superfields, which we do not consider in this work. If that was not the case one should take into account the gauge symmetry of the higher spin (super)fields. The result of that would be that, the spectrum of the half integer supermultiplet will collapse from $(s+1, s+1 / 2, s+1 / 2, s)$ to $(s+1, s+1 / 2)$ and more importantly the generator of cubic interactions $\mathcal{J}_{\alpha(s) \dot{\alpha}(s)}$ in (3.5) will become conserved higher spin supercurrents. Such supercurrents have been found in [68-76] and their structure is consistent with (3.7).
} 
fields required by supersymmetry in order to balance the bosonic and fermionic degrees of freedom and also make the symmetry manifest. However, these auxiliary fields do not have any dynamics and can be integrated out. By doing so, we obtain an effective theory with on-shell supersymmetry which includes two copies of the previously discussed bosonic higher spin interactions. That is because there are two higher spin bosons, one with spin $s$ and one with spin $s+1$. Additionally, we obtain terms that depend on the higher spin fermions $\left(\psi_{\alpha(s+1) \dot{\alpha}(s)}, \xi_{\alpha(s+1) \dot{\alpha}(s)}\right)$ and the 'inflatino' $\left(\chi_{\alpha}\right)$. The interactions are given by,

$$
\begin{aligned}
\mathcal{L}_{\text {Bosonic }}= & h^{\alpha(s+1) \dot{\alpha}(s+1)}\left[\frac{\lambda_{s+1}}{\Lambda^{s-2}} \partial^{(s+1)} \zeta+\sum_{p=0}^{s+1} \frac{\kappa_{p}^{s+1}}{\Lambda^{s-2}} \partial^{(p)} \zeta \partial^{(s+1-p)} \zeta+\ldots\right] \\
& +h^{\alpha(s) \dot{\alpha}(s)}\left[\frac{\lambda_{s}}{\Lambda^{s-3}} \partial^{(s)} \zeta+\sum_{p=0}^{s} \frac{\kappa_{p}^{s}}{\Lambda^{s-3}} \partial^{(p)} \zeta \partial^{(s-p)} \zeta+\ldots\right] \\
\mathcal{L}_{\text {Fermionic }}= & \psi^{\alpha(s+1) \dot{\alpha}(s)}\left[\frac{r_{s}}{\Lambda^{s-1}} \partial^{(s)} \chi_{\alpha}+\frac{\lambda_{s}}{\Lambda^{s-1}} \chi_{\alpha} \partial^{(s)} \zeta+\sum_{p=1}^{s} \frac{v_{p}}{\Lambda^{s-1}} \partial^{(p)} \chi \partial^{(s-p)} \zeta+\ldots\right]+\text { h.c. } \\
& +\xi^{\alpha(s+1) \dot{\alpha}(s)}\left[\frac{t_{s}}{\Lambda^{s-1}} \partial^{(s)} \chi_{\alpha}+\frac{\lambda_{s}}{\Lambda^{s-1}} \chi_{\alpha} \partial^{(s)} \zeta+\sum_{p=1}^{s} \frac{w_{p}}{\Lambda^{s-1}} \partial^{(p)} \chi \partial^{(s-p)} \zeta+\ldots\right]+\text { h.c. }
\end{aligned}
$$

where ... indicates additional and higher-order terms.

As discussed in section 2, on-shell supersymmetry should be preserved only in the higher spin sector, and not in inflaton sector which breaks supersymmetry with the inflationary vacuum energy $H^{2}$. In our effective Lagrangian this information can be entered by hand by removing any correlation between the coupling constants of the inflatino and those of the inflaton. For example we can assign the inflatino $\chi$ a mass $m_{\chi} \gtrsim H$. Typically one would then integrate out the inflatino, thereby eliminating all the contributions at linear order in the higher spin fermions (3.9). However it is the inclusion of such heavy fields that we are explicitly after in this work. Indeed, the higher spin fields themselves have mass $m>\sqrt{s(s-1)} H$ by the Higuchi bound [66, 67]. The breaking of supersymmetry will also induce differing loop corrections to the on-shell couplings of $\zeta$ to bosonic (3.8) and fermionic (3.9) higher spin fields. Depending on the precise details of the model, there may also be classical corrections to these parameters, for example, from a quartic interaction involving an additional scalar field that gains a VEV in the SUSY-breaking vacuum. For our analysis, we assume for simplicity that there are no such classical corrections, and that these couplings are equal at tree level. This does not alter the analysis in any way other then the overall prefactor of the result.

To make contact with the framework of effective field theories within we have to work, as presented in section 2, we must break the time translations part of the Poincare group and write interaction terms which include fermionic higher spin particles up to linear order. From equation (3.9), and taking into account contributions coming from the $\sqrt{-g}$ part of the action, one has to consider the following fermionic interaction Lagrangian for a spin- 
$s+1 / 2$ field:

$$
\mathcal{L} \supset \frac{\lambda_{s}}{\Lambda^{s-1}} \partial_{i_{1} \ldots i_{s}} \zeta \bar{\chi} \psi^{i_{1} \ldots i_{s}}+\frac{g_{s}}{\Lambda^{s}} \dot{\zeta} \partial_{i_{1} \ldots i_{s}} \zeta \bar{\chi} \psi^{i_{1} \ldots i_{s}}+\frac{\kappa_{s}}{\Lambda^{s}} \dot{\zeta} \partial_{i_{1} \ldots i_{s}} \bar{\chi} \psi^{i_{1} \ldots i_{s}}+c . c .
$$

where the coupling to $\dot{\zeta}$ enters from the metric perturbation $\delta g^{00}$, as in equation (2.13). The full fermionic interaction Lagrangian is composed of copies of (3.10) for the appropriate fermions in the supermultiplet. Armed with this, we can now return to the cosmological collider.

\section{Higher spin supersymmetry at the cosmological collider}

The statistical correlations of temperature fluctuations in the cosmic microwave background descend from the initial conditions prepared for it by inflation. This can be computed via the Schwinger-Keldysh formalism, colloquially called the 'In-In' formalism, in which the choice of integration contour allows for ignorance as to the future evolution of the universe. Introduced to cosmology in [77], there are now many excellent reviews on this topic, see e.g. [78-80], and see [81] for a textbook treatment of the field theory aspects.

Correlation functions can be computed in this framework by splitting the Hamiltonian into a free Hamiltonian $H_{0}$ and interaction Hamiltonian $H_{\text {int }}$. From this, one can define the interaction picture fields as having propagator determined solely by $H_{0}$, and correlation functions of operators built from the full fields can be computed as contractions of the interaction picture fields with the interaction Hamiltonian.

More precisely, the expectation value of an operator $W$ is given by,

$$
\langle W(t)\rangle=\left\langle\left[\overline{\mathrm{T}} e^{i \int_{-\infty(1-i \epsilon)}^{t} H_{\mathrm{int}}^{I}\left(t^{\prime}\right) \mathrm{d} t^{\prime}}\right] W^{I}(t)\left[\mathrm{T} e^{-i \int_{-\infty(1+i \epsilon)}^{t} H_{\mathrm{int}}^{I}\left(t^{\prime \prime}\right) \mathrm{d} t^{\prime \prime}}\right]\right\rangle,
$$

where $H_{\text {int }}^{I}$ and $W^{I}$ are the interaction Hamiltonian and the operator $W$ built out of interaction picture fields. The simplest quantity one can compute from this is the expectation value given a single insertion of the interaction Hamiltonian. In that case, the above expression reduces to

$$
\langle W(t)\rangle=-2 \operatorname{Re}\left\langle i W^{I}(t) \int_{-\infty(1-i \epsilon)}^{t} H_{\mathrm{int}}^{I}\left(t^{\prime}\right) \mathrm{d} t^{\prime}\right\rangle+\ldots,
$$

where the ... corresponds to additional insertions of $H_{\mathrm{int}}$. For the case of the curvature perturbation 3-point function, $W=\zeta^{3}$, this picks out the intrinsic non-Gaussianity, first computed in [77]. This is the 3-point function induced by the self-interactions of $\zeta$ in an inflationary background. The result is given by

$$
\begin{aligned}
\left\langle\zeta\left(k_{1}, 0\right) \zeta\left(k_{2}, 0\right) \zeta\left(k_{3}, 0\right)\right\rangle= & (2 \pi)^{3} \delta\left(k_{1}+k_{2}+k_{3}\right) \frac{H^{4}}{m_{p l}^{4}} \frac{1}{\left(k_{1} k_{2} k_{3}\right)^{3}} \frac{1}{4 \epsilon^{2}} \\
& \cdot\left[\frac{\eta}{8} \sum k_{i}^{3}+\frac{\epsilon}{8}\left(-\sum k_{i}^{3}+\sum_{i \neq j} k_{i} k_{j}^{2}+\frac{8}{k_{1}+k_{2}+k_{3}} \sum_{i>j} k_{i}^{2} k_{j}^{2}\right)\right] .
\end{aligned}
$$


This is typically expressed in the limit that one of the momenta is much smaller than the other two, in what is referred to as 'squeezed limit'. The result then takes a simplified form

$$
\lim _{k_{1} \rightarrow 0}\left\langle\zeta\left(k_{1}, 0\right) \zeta\left(k_{2}, 0\right) \zeta\left(k_{3}, 0\right)\right\rangle=(2 \pi)^{3} f_{N L} \delta\left(k_{1}+k_{2}+k_{3}\right) \frac{H^{4}}{16 \epsilon^{2} m_{p l}^{4}} \frac{1}{\left(k_{1} k_{2} k_{3}\right)^{3}} \sum k_{i}^{3},
$$

which corresponds to 'local shape' non-Gaussianity [82] with amplitude $f_{N L}$ given by

$$
f_{N L}=\frac{\eta}{2}+\epsilon .
$$

The inflationary slow-roll conditions $\epsilon, \eta \ll 1$ thus imply the intrinsic non-Gaussianity in single-field slow-roll inflation is extremely small, $f_{N L} \ll 1$.

Additional insertions of $H_{\text {int }}$ capture the effect of particle exchange. Given the slowroll suppression of the intrinsic non-Gaussianity, this can easily be the dominant effect. It is in this sense that CMB non-Gaussianity is a particle detector, with inflation as the cosmological collider.

In this work we are concerned with the non-Gaussianity induced via the exchange of a higher spin particle, as described by the insertion of two interaction Hamiltonians. This is captured by the quadratic terms in the expansion of (4.1), see e.g. [83],

$$
\langle W(t))\rangle=\int \mathrm{d} t^{\prime} \int \mathrm{d} t^{\prime \prime}\left\langle H^{I}\left(t^{\prime}\right) W(t) H^{I}\left(t^{\prime \prime}\right)\right\rangle-2 \operatorname{Re} \int \mathrm{d} t^{\prime} \int \mathrm{d} t^{\prime \prime}\left\langle W(t) H^{I}\left(t^{\prime}\right) H^{I}\left(t^{\prime \prime}\right)\right\rangle,
$$

with appropriate $i \epsilon$ prescriptions in the integrations.

\subsection{Effective action and relevant interactions}

We consider an effective action describing the interactions of a scalar $\zeta$, a massive spin$1 / 2$ field $\chi$, and the propagating component fields of the massive, half-integer superspin $\mathrm{Y}=s+1 / 2$ supermultiplet, as they have been discussed previously. We consider our action as an expansion in higher spin fields, keeping up to linear order terms. We consider

$$
\mathcal{L}=\mathcal{L}_{\zeta}+\mathcal{L}_{\chi}+\mathcal{L}_{h s}+\mathcal{O}\left(h s^{2}\right) \ldots
$$

In this work we are particularly interested in the impact of the fermionic higher spin particles, which has thus far been left unstudied. Motivated from the discussion in section 3 and (3.10), we take the Lagrangian of one fermionic spin- $s+1 / 2$ particle $\psi$ interacting with a dimensionless scalar $\zeta$ and the spin- $1 / 2$ particle $\chi$,

$$
\mathcal{L} \supset \frac{\lambda_{s}}{\Lambda^{s-1}} \partial_{i_{1} \ldots i_{s}} \zeta \bar{\chi} \psi^{i_{1} \ldots i_{s}}+\frac{g_{s}}{\Lambda^{s}} \dot{\zeta} \partial_{i_{1} \ldots i_{s}} \zeta \bar{\chi} \psi^{i_{1} \ldots i_{s}}+\text { c.c. },
$$

where $\lambda_{s}$ and $g_{s}$ are dimensionless coupling constants, $\Lambda$ is a UV cutoff, and fermionic indices are contracted between $\bar{\chi}$ and $\psi$. For simplicity we have taken $\kappa_{s}=0$ in (3.10), which leads to the same angular dependence for $\langle\zeta \zeta \zeta\rangle$ as the two terms above. The relevant Feynman diagrams, or rather their equivalent in the Schwinger-Keldysh ("in-in" formalism) are shown in figure 1. 


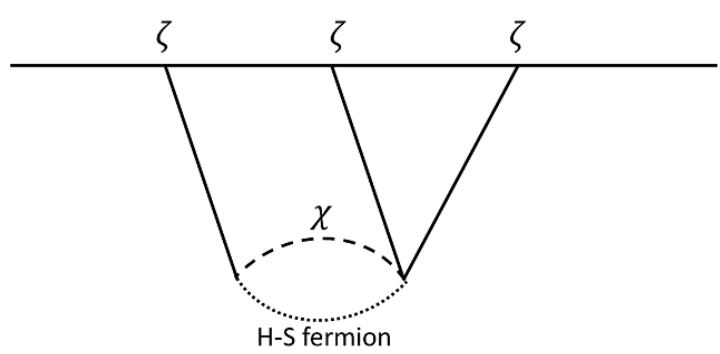

(a) Exchange of a higher spin Fermion

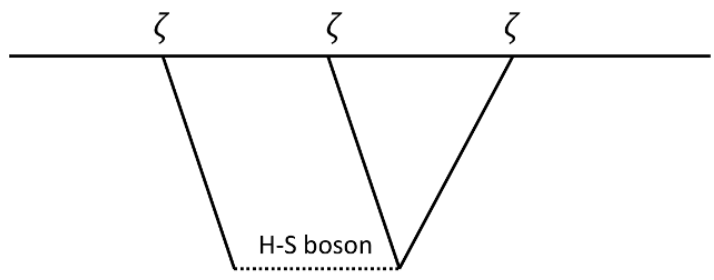

(b) Exchange of a higher spin boson

Figure 1. In-In formalism Feynman Diagrams for exchange of a single higher spin particle.

\subsection{Higher spin fields in de Sitter space}

To evaluate the three-point function in the Schwinger-Keldysh formalism, we first must have expressions for the free fields in de Sitter space.

To begin with, a scalar field $\phi$ is quantized in curved space as,

$$
\phi(x, t)=\int \frac{\mathrm{d}^{3} k}{(2 \pi)^{3}} \phi_{k} a_{k} e^{i k \cdot x}+\text { h.c. . }
$$

The coefficients $\phi_{k}(k, t)$ are referred to as "mode functions". The field $\phi(x)$ and mode functions $\phi_{k}$ are related to two-point correlation functions as follows. The position-space two point function of a free-field $\phi$ is given by,

$$
\langle\phi(x) \phi(y)\rangle=\int \frac{\mathrm{d}^{3} k}{(2 \pi)^{3}}\left\langle\phi_{k} \phi_{k}\right\rangle e^{i k(x-y)}=\int \mathrm{d} \log k \frac{k^{3}}{2 \pi^{2}}\left|\phi_{k}\right|^{2} e^{i k(x-y)} \equiv \int \mathrm{d} \log k \Delta_{\phi}^{2}(k) e^{i k(x-y)} .
$$

The last equality defines the dimensionless power spectrum, $\Delta_{\phi}^{2}(k) \equiv \frac{k^{3}}{2 \pi^{2}}\left|\phi_{k}\right|^{2}$. A special case of the above is a scale-invariant spectrum. In this case, $\Delta_{\phi}^{2}(k)$ is a constant, which owes its name to the implied scaling symmetry of the two-point function, $\langle\phi(x) \phi(y)\rangle=$ $\langle\phi(\lambda x) \varphi(\lambda y)\rangle$. During inflation, this scaling symmetry of a massless scalar has its origins in the dilatation symmetry at late times in de Sitter space.

An important example is the curvature perturbation on uniform density hypersurfaces, $\zeta$. This has mode function given by,

$$
\zeta_{k} \simeq \frac{H}{m_{p l} \sqrt{4 \epsilon k^{3}}}(1-i k \eta) e^{i k \eta}
$$

where $\epsilon=-\dot{H} / H^{2} \ll 1$ is the inflationary slow-roll parameter, and $\eta$ is conformal time $\mathrm{d} \eta \equiv a^{-1} \mathrm{~d} t$, which in de Sitter space is given by $\eta=-1 /(a H)$. This solution is found by explicitly solving the Klein-Gordon equation in de Sitter space. Inflation is a small deviation from de Sitter space, which converts the scaling with $k$ to $k^{-3 / 2+\left(n_{s}-1\right) / 2}$, where $n_{s}$ defines the spectral index of the power spectrum,

$$
\Delta_{\zeta}^{2} \equiv \frac{k^{3}}{2 \pi^{2}}\left|\zeta_{k}\right|^{2} \propto k^{n_{s}-1}
$$


Another important example is massless spin-2, e.g. the graviton. Expanded in helicity states $\lambda= \pm 2$, the mode functions are given by [54]

$$
\gamma_{k}^{\lambda}=\frac{\sqrt{2} H}{m_{p l}} \frac{1}{\sqrt{k^{3}}}(1+i k \eta) e^{-i k \eta},
$$

which, importantly, differs from the curvature perturbation (4.11) in part by an factor of $1 / \sqrt{\epsilon}$. The power spectrum of primordial gravitational waves on large scales $k \eta \rightarrow 0$ is then given by [54],

$$
\Delta_{\gamma}^{2} \equiv \sum_{\lambda} \frac{k^{3}}{2 \pi^{2}}\left|\gamma_{k}^{\lambda}\right|=\frac{2}{\pi^{2}} \frac{H^{2}}{m_{p l}^{2}},
$$

which is a direct probe of the energy scale of inflation [84]. The ratio of the tensor power spectrum (4.14) to scalar power spectrum, termed the 'tensor-to-scalar ratio', is given by

$$
r \equiv \frac{\Delta_{\gamma}^{2}}{\Delta_{\zeta}^{2}}=16 \epsilon
$$

where again $\epsilon \ll 1$ is the inflationary slow-roll parameter.

In contrast with these two examples, for massive particles the two-point function and hence mode functions are suppressed on large scales and at late times. For a minimallycoupled massive scalar field $\sigma$, the two-point function has the exact solution [85]

$$
\left\langle\sigma_{k}(\eta) \sigma_{k}\left(\eta^{\prime}\right)\right\rangle=\frac{\pi}{4} H^{2}\left(\eta \eta^{\prime}\right)^{3 / 2} e^{-\pi \mu} H_{i \mu}^{(1)}(-k \eta) H_{i \mu}^{(1) *}\left(-k \eta^{\prime}\right),
$$

where $\mu \equiv \sqrt{m / H^{2}-9 / 4}$, and $H_{i \mu}^{(1)}$ is the Hankel function of the first kind. This corresponds to a mode function $\sigma_{k} \sim H \eta^{3 / 2} e^{-\pi \mu / 2}=a(\eta)^{-3 / 2} e^{-\pi \mu / 2} / \sqrt{H}$, the latter equality using $\eta=-1 / a H$ during inflation.

Now we turn to massive particles with spin. These are constrained by the Higuchi bound to have mass satisfying $m^{2} \geq s(s-1) H^{2}$. As for scalars, the isometries of dS fixes the scaling of the two-point correlation function of spinning fields [12], which takes the form

$$
\left\langle\mathcal{O}_{s}(k) \mathcal{O}_{s}(k)\right\rangle \propto k^{2 \Delta-3},
$$

where all Lorentz indices are contracted with $s$ copies of a null vector, and $\Delta$ is the scaling dimension of the field,

$$
\Delta=\frac{3}{2}-i \mu_{s}, \quad \mu_{s}=\sqrt{\frac{m^{2}}{H^{2}}-\left(s-\frac{1}{2}\right)^{2}} .
$$

For heavy fields, or more precisely the "principle series" [85], one has $\operatorname{Re} \Delta=3 / 2$, and the mode function is simply

$$
\left\langle\mathcal{O}_{s}(k) \mathcal{O}_{s}(k)\right\rangle \propto k^{-2 i \mu_{s}} .
$$

The prefactors follow from dimensional analysis, and intuition from solving the KleinGordon equation for heavy fields, which leads to an additional $e^{-\pi \mu_{s}}$ suppression. Importantly, this applies to general operators with spin, and not to just to bosonic (integer 
spin) operators, but to fermionic (half-integer spin) as well, and the two-point function of half-integer operators is similarly constrained to scale as $k^{2 \Delta-3}$ as in (4.17).

In this work we will focus on the angular dependence of correlation functions. Given this, for simplicity we ignore the $e^{i \mu_{s}}$ phase, though we note that this can lead to oscillations in $k$-space [13], and thus is of potential interest. Dropping this phase, the mode functions for spin- $s$ and spin- $(s+1 / 2)$ are at late times given by

$$
\mathcal{O}_{s}(k, \eta) \simeq \frac{a(\eta)^{-3 / 2}}{\sqrt{H}} e^{-\pi \mu_{s} / 2}, \mathcal{O}_{s+1 / 2}(k, \eta) \simeq a(\eta)^{-3 / 2} e^{-\pi \mu_{s+1 / 2} / 2} .
$$

The scaling with $a(\eta)$ follows from solving the mode-function equation of motion explicitly, ${ }^{9}$ while the differing factors of $H$ follow from dimensional analysis. ${ }^{10}$ This matches with the known result for a heavy spin-1/2 particle in de Sitter space [86]: a spin- $1 / 2$ fermion $\chi$ with mass $m>H$ has mode function given by,

$$
\chi_{k} \simeq a(\eta)^{-3 / 2} e^{-\pi m_{\chi} / 2 H} .
$$

Now we can make this more precise. A massive spin-s boson may be split into helicity components as,

$$
\sigma_{\mu_{1} \ldots \mu_{s}}=\sum_{\lambda=-s}^{s} \sigma_{\mu_{1} \ldots \mu_{s}}^{\lambda}
$$

and then decomposed into fields of $n$ polarization directions by projecting the spinning field $\sigma_{\mu_{1} \ldots \mu_{s}}$ onto spatial slices, i.e. via the decomposition

$$
\sigma_{i_{1} \ldots i_{n} \eta \ldots \eta}=\sum_{\lambda} \sigma_{n, s}^{\lambda} \varepsilon_{i_{1} \ldots i_{n}}^{\lambda}
$$

where $\eta$ is the time coordinate. Here, the $s$ index refers to the spin, $n$ refers to the 'spatial spin,' and $\lambda$ is the helicity of the field. Thus $\varepsilon_{i_{s} \ldots i_{n}}^{\lambda}$ is a normalized, totally symmetric tensor with spin $s$ and helicity $\lambda$. The $\sigma_{n, s}^{\lambda}$ satisfy $\sigma_{n, s}^{\lambda}=0$ for $n<|\lambda|$ [13].

The quantity that appears in scattering with scalars is $\lambda=0$ and $n=s$ (for more details see [13] or appendix A), i.e. the quantity $\sigma_{s, s}^{0}$. Explicitly solving for the mode function, one finds [13]

$$
\sigma_{s, s}^{0}(k, \eta) \simeq \frac{a(\eta)^{s-3 / 2}}{\sqrt{H}} e^{-\pi \mu_{s} / 2}
$$

\footnotetext{
${ }^{9}$ In solving the Klein-Gordon equation explicitly, the scaling with $a(\eta)$ depends on the number of upper vs. lower indices, but this dependence cancels when all indices are contracted insider of correlation functions. For example, mode functions defined with respect to $\mathcal{O}_{s}$ with all lower indices are

$$
\mathcal{O}_{i_{1} \ldots i_{s}}(k, \eta) \propto \frac{a(\eta)^{s-3 / 2}}{\sqrt{H}}
$$

See (B.76) and (C.7) of [85]: using $\eta=1 /(a H)$ and $N_{\lambda}=(1 / \sqrt{k})(k / H)^{s-1}$, one finds that $N_{\lambda}(k \eta)^{3 / 2-s}=$ $(1 / \sqrt{k})(k / H)^{s-1}(k / a H)^{3 / 2-s}=a^{s-3 / 2} / \sqrt{H}$.

${ }^{10}$ Bosons are defined as having mass term $m^{2}\left(\mathcal{O}^{b}(x)\right)^{2}$ and hence mass dimension 1 while fermions are defined as having mass term $m \overline{\mathcal{O}}^{f}(x) \mathcal{O}^{f}(x)$ and hence mass dimension $3 / 2$. The corresponding mode functions have dimension $-1 / 2$ and 0 .
} 
Moreover, for the above $\lambda=0$ helicity state, one has the important relation,

$$
\hat{q}_{i_{1}} \hat{q}_{i_{2}} \ldots \hat{q}_{i_{s}} \varepsilon_{i_{1} \ldots i_{s}}^{\lambda=0}(\hat{k}, \varepsilon)=P_{s}(\cos \theta),
$$

with $\theta$ defined as the angle between $\hat{q}$ and $\hat{k}$. This follows from more general relations for spin- $s$ polarization vectors, which are detailed in the thesis [85], and given in appendix A.

Similar to the bosonic case, a massive spin- $(s+1 / 2) 4$-component fermion may be split into helicity components as,

$$
\psi_{\mu_{1} \ldots \mu_{s}}^{\alpha}=\sum_{\lambda} \psi_{\mu_{1 \ldots \mu_{s}}^{\lambda \alpha}}^{\lambda \alpha}
$$

where $\alpha$ is a fermionic index and $\mu_{1} \ldots \mu_{s}$ are bosonic indices, and projected onto spatial slices via the decomposition,

$$
\psi_{i_{1} \ldots i_{n} \eta \ldots \eta}^{\alpha}=\sum_{\lambda} \psi_{n, s}^{\lambda} \epsilon_{i_{1} \ldots i_{n}}^{\lambda \alpha}
$$

where again $\eta$ is the time coordinate. We construct the spin- $(s+1 / 2)$ polarization vectors as a tensor product of spin- $1 / 2$ and spin- $s$. That is, we decompose,

$$
\epsilon_{i_{1} \ldots i_{s}}^{\lambda \alpha}=\sum_{\lambda^{\prime}} \xi_{\lambda^{\prime}}^{\alpha} \varepsilon_{i_{1} \ldots i_{s}}^{\lambda}
$$

with $\xi_{\lambda^{\prime}}$ a spin-1/2 eigenspinor of helicity $\lambda^{\prime}$ and $\varepsilon^{\lambda}$ the spin- $s$ polarization vector of helicity $\lambda$. The general decomposition of the fermion field can then be written as,

$$
\psi_{i_{1} \ldots i_{n} \eta \ldots \eta}^{\alpha}=\sum_{\lambda, \lambda^{\prime}} \psi_{n, s}^{\lambda \lambda^{\prime}} \epsilon_{i_{1} \ldots i_{n}}^{\lambda} \xi^{\lambda^{\prime} \alpha}
$$

Similar to the bosonic case, the fermions can be split into helicity states, and it is the helicity $0 \pm 1 / 2$ which contributes to the loop in figure 1a. More explicitly, the relevant mode function has the form,

$$
\psi_{s, s}^{0 \lambda^{\prime}} \simeq a(\eta)^{s-3 / 2} e^{-\pi \mu_{s} / 2} .
$$

One can use this, along with the mode functions (4.22) and (4.11), and the interaction Lagrangian (4.8), to compute correlation functions of $\zeta$ involving intermediate states of fermions.

\subsection{Non-Gaussianity from higher spin particle exchange}

The correlator we wish to compute is of three $\zeta(k, \eta)$ at lates times, $\eta \rightarrow 0$, and in the limit that one of the momenta is small $k_{1} \ll k_{2}, k_{3}$, i.e. the quantity,

$$
\left\langle\zeta\left(k_{1}, 0\right) \zeta\left(k_{2}, 0\right) \zeta\left(k_{3}, 0\right)\right\rangle,
$$

given the insertion of interaction vertices between the scalar $\zeta$ and higher spin fields. The result for exchange of a higher spin boson are given in [12, 13]. While [12] focused on the scaling and angular dependence, [13] explicitly solved the Klein-Gordon equation for higher spin bosonic fields and from this was able to compute all expressions exactly. In our analysis we will follow [12] and focus on the amplitude and angular dependence. 
The 3-point function resulting from higher spin boson exchange, diagram $1 \mathrm{~b}$, is given by [13],

$\lim _{k_{1} \ll k_{3}, \eta \rightarrow 0} \frac{\left\langle\zeta\left(k_{1}\right) \zeta\left(k_{2}\right) \zeta\left(k_{3}\right)\right\rangle}{\Delta_{\zeta}^{4}}=\alpha_{s} \Delta_{\zeta}^{-1} \times P_{s}\left(\hat{k}_{1} \cdot \hat{k}_{3}\right) \times \mathcal{I}^{(s)}\left(\mu_{s}, c_{\pi}, k_{1}, k_{3}, k_{3}\right) \delta\left(\sum k_{i}\right)+\left(k_{2} \leftrightarrow k_{3}\right)$,

where $\mathcal{I}^{(s)}\left(\mu_{s}, c_{\pi}, k_{1}, k_{3}, k_{3}\right)$ is a complicated function of momenta given in the appendices of [13], and $P_{s}$ the Legendre polynomial. This is characterized by a dimensionless coupling $\alpha_{s}$, which in the notation of our (2.13), and taking the Goldstone boson parameters of [13] to be $c_{\pi}=1$ and $f_{\pi}=m_{p l}$, is given by,

$$
\alpha_{s}=\lambda_{s} g_{s}\left(\frac{\Lambda}{m_{p l}}\right)^{6}\left(\frac{H}{\Lambda}\right)^{2 s+1} .
$$

This corresponds to a non-Gaussianity parameter of

$$
f_{\mathrm{NL}} \equiv \frac{5}{18} \frac{\left\langle\zeta_{k} \zeta_{k} \zeta_{k}\right\rangle}{P_{\zeta}(k)^{2}} \sim e^{-\pi \mu_{s}} \alpha_{s} \Delta_{\zeta}^{-1}
$$

with shape function

$$
\lim _{k_{1} \ll k_{3}}\left\langle\zeta\left(k_{1}, 0\right) \zeta\left(k_{2}, 0\right) \zeta\left(k_{3}, 0\right)\right\rangle \propto \frac{1}{k_{1}^{3} k_{3}^{3}}\left(\frac{k_{1}}{k_{3}}\right)^{2},
$$

and a characteristic angular dependence,

$$
\left\langle\zeta\left(k_{1}, 0\right) \zeta\left(k_{2}, 0\right) \zeta\left(k_{3}, 0\right)\right\rangle \propto P_{s}(\cos \theta),
$$

with $\theta$ the angle between $k_{1}$ and $k_{3}$.

We now turn to the fermions. Before we proceed, it is important to further clarify and emphasize the procedure. We approximate the fermionic mode functions by their super-horizon scaling (4.31), which neglects the sub-horizon oscillatory behaviour of the exact solution (see [13] for the bosonic results). This is sufficient to compute the angular dependence of the 3 -point function, as is our aim, but not the $k$-dependence and thus not the shape function. Additionally, we regulate the fermionic loop of figure 1a by imposing a UV cutoff, which we choose to be the Hubble scale for self-consistency with our approximate form of the mode functions. The choice of cutoff does not qualitatively affect the result, and can be undone by a simple replacement $H \rightarrow \Lambda_{U V}$ in the loop integral.

To compute the fermion diagram figure 1a there are two interaction Hamiltonians, which follow from the Lagrangian (4.8), given by

$$
H_{\mathrm{int} 1}=\frac{\lambda_{s}}{\Lambda^{s-1}} \int d^{3} x \frac{1}{a^{2 s-3}} \partial_{i_{1} \ldots i_{s}} \zeta \bar{\chi} \psi_{i_{1} \ldots i_{s}}+\text { h.c. },
$$

and

$$
H_{\mathrm{int} 2}=\frac{g_{s}}{\Lambda^{s}} \int d^{3} x \frac{1}{a^{2 s-2}} \zeta^{\prime} \partial_{i_{1} \ldots i_{s}} \zeta \bar{\chi} \psi_{i_{1} \ldots i_{s}}+\text { h.c. }
$$


with $i$ indices summed over, and where ' indicates a derivative with respect to conformal time. The 3-point function of three $\zeta(k, \eta)$, at late times $\eta \rightarrow 0$, is given by

$$
\left\langle\zeta\left(k_{1}, 0\right) \zeta\left(k_{2}, 0\right) \zeta\left(k_{3}, 0\right)\right\rangle=\operatorname{Re} 4\left\langle\left[\zeta\left(k_{1}, 0\right) \zeta\left(k_{2}, 0\right) \zeta\left(k_{3}, 0\right) \int a\left(\eta^{\prime}\right) \mathrm{d} \eta^{\prime} H_{\text {int } 1}\left(\eta^{\prime}\right) \int a\left(\eta^{\prime \prime}\right) \mathrm{d} \eta^{\prime \prime} H_{\text {int } 2}\left(\eta^{\prime \prime}\right)\right]\right\rangle .
$$

where we focus on the second term in (4.6), which has the same angular dependence as the first term, the two terms differing in calculation only by the distribution of complex conjugation among the resulting $\zeta$ mode functions. The additional factor of 2 as compared to (4.6) is due to the two possibilities of time-ordering $H_{1}$ and $H_{2}$.

To compute this we expand $H_{\text {int1 }}$ and $H_{\text {int2 }}$ in momentum space, which results in 7 momentum integrals, $\mathrm{d}^{3} q_{1} \ldots \mathrm{d}^{3} q_{7}$. We define the momenta as follows: let $k_{1}$ be the $\zeta$ in the left interaction vertex of figure 1a, and $\ell$ the momentum of $\chi$ in the loop and $\ell+k_{1}$ the momentum of $\psi$ in the loop. The 'outgoing' $\zeta$ momenta are defined as $k_{2}$ and $k_{3}$; under Wick contractions we will need to sum over $k_{2} \rightarrow k_{3}$.

This gives, approximating the mode functions by their forms given in section 4.2 ,

$$
\begin{aligned}
& \left\langle\zeta\left(k_{1}, 0\right) \zeta\left(k_{2}, 0\right) \zeta\left(k_{3}, 0\right)\right\rangle \\
& =\frac{\lambda_{s} g_{s}}{\Lambda^{2 s-1}} \operatorname{Re} 8 \zeta_{k_{1}}(0) \zeta_{k_{2}}(0) \zeta_{k_{3}}(0) \int \frac{\mathrm{d} \eta^{\prime}}{a\left(\eta^{\prime}\right)^{s-1}} \int \frac{\mathrm{d} \eta^{\prime \prime}}{a\left(\eta^{\prime \prime}\right)^{s}} \zeta_{k_{1}}^{*}\left(\eta^{\prime}\right) \zeta_{k_{2}}^{*}\left(\eta^{\prime \prime}\right) \zeta_{k_{3}}^{*}\left(\eta^{\prime \prime}\right) \\
& \quad \cdot\left|k_{1}\right|^{s}\left|k_{3}\right|^{s} e^{-\pi \mu_{s}} e^{-\pi m_{\chi} / H} \delta\left(\sum k_{i}\right) \\
& \quad \cdot \int \frac{\mathrm{d}^{3} \ell}{(2 \pi)^{3}} P_{s}\left(\hat{k}_{1} \cdot \hat{q}\right) P_{s}\left(\hat{k}_{3} \cdot \hat{q}\right)\left(\sum_{\lambda} \xi^{\lambda}(\hat{\ell}) \xi_{\lambda}^{\dagger}(\hat{q})\right)^{2}+k_{2} \leftrightarrow k_{3},
\end{aligned}
$$

where $\vec{q} \equiv \vec{\ell}+\overrightarrow{k_{1}}$. We can factorize this into three pieces:

$$
\left\langle\zeta\left(k_{1}, 0\right) \zeta\left(k_{2}, 0\right) \zeta\left(k_{3}, 0\right)\right\rangle \equiv \operatorname{Re} \mathcal{I}_{1} \mathcal{I}_{2} \mathcal{I}_{3}
$$

with time-integrals,

$$
\mathcal{I}_{1} \equiv \zeta_{k_{1}}(0) \zeta_{k_{2}}(0) \zeta_{k_{3}}(0) \int \frac{\mathrm{d} \eta^{\prime}}{a\left(\eta^{\prime}\right)^{s-1}} \int \frac{\mathrm{d} \eta^{\prime \prime}}{a\left(\eta^{\prime \prime}\right)^{s}} \zeta_{k_{1}}^{*}\left(\eta^{\prime}\right) \zeta_{k_{2}}^{*}\left(\eta^{\prime \prime}\right) \zeta_{k_{3}}^{*}\left(\eta^{\prime \prime}\right),
$$

momentum integrals,

$$
\mathcal{I}_{2} \equiv \int \frac{\mathrm{d}^{3} \ell}{(2 \pi)^{3}} P_{s}\left(\hat{k}_{1} \cdot \hat{q}\right) P_{s}\left(\hat{k}_{3} \cdot \hat{q}\right)\left(\sum_{\lambda} \xi^{\lambda}(\hat{\ell}) \xi_{\lambda}^{\dagger}(\hat{q})\right)^{2},
$$

and an overall prefactor of

$$
\mathcal{I}_{3} \equiv \frac{\lambda_{s} g_{s}}{\Lambda^{2 s-1}} 8\left|k_{1}\right|^{s}\left|k_{3}\right|^{s} e^{-\pi \mu_{s}} e^{-\pi m_{\chi} / H} \delta\left(\sum k_{i}\right) .
$$

The loop integral $\mathcal{I}_{2}$ is UV-divergent, and we apply a cutoff at $H$,

$$
\mathcal{I}_{2} \simeq H^{3} \int \mathrm{d} \Omega P_{s}\left(\hat{k}_{1} \cdot \hat{q}\right) P_{s}\left(\hat{k}_{3} \cdot \hat{q}\right)\left(\sum_{\lambda} \xi^{\lambda}(\hat{\ell}) \xi_{\lambda}(\hat{q})\right)^{2},
$$


with $\hat{q}$ now given by

$$
\hat{q}=\frac{H \hat{\ell}+k_{1} \hat{k}_{1}}{\sqrt{H^{2}+k_{1}^{2}}} \simeq \hat{\ell}
$$

where the latter equality follows working in the limit $k_{1} \rightarrow 0$. This simplifies the sum over spin- $1 / 2$ helicities, as the $\xi(\hat{k})$ are normalized to 1 . Thus we have,

$$
\mathcal{I}_{2} \simeq H^{3} \int \frac{\mathrm{d} \Omega}{\left(2 \pi^{3}\right)} P_{s}\left(\hat{k}_{1} \cdot \hat{q}\right) P_{s}\left(\hat{k}_{3} \cdot \hat{q}\right)
$$

The remaining integral over angles can be performed analytically. Defining $k_{1}$ as making angle $\theta_{1}=0$ in the $\{x, y\}$ plane, and $k_{3}$ as making angle $\theta_{13}$, such that $\hat{k}_{1} \cdot \hat{k}_{3}=\cos \theta_{13}$, $\hat{q} \cdot \hat{k}_{1}=\cos \theta, \hat{q} \cdot k_{3}=\cos \left(\theta-\theta_{13}\right)$, the integral can be written as,

$$
\mathcal{I}_{2} \simeq \frac{H^{3}}{8 \pi^{2}} \int \mathrm{d} \cos \theta P_{s}(\cos \theta) P_{s}\left(\cos \left(\theta-\theta_{13}\right)\right)
$$

We then use the identity, ${ }^{11}$

$$
P_{\ell}(\cos (a-b))=\sum_{m=-\ell}^{\ell} P_{\ell}^{m}(\cos a) P_{\ell}^{m}(\cos b) \frac{(\ell-m) !}{(\ell+m) !}
$$

from which one can evaluate the integral explicitly. The result is

$$
\mathcal{I}_{2}=\frac{H^{3}}{8 \pi^{2}} \sum_{m=-s}^{s} c_{m} P_{s}^{m}\left(\cos \theta_{13}\right)
$$

with coefficients

$$
c_{m}=\frac{(s-m) !}{(s+m) !} \int_{-1}^{1} \mathrm{~d} x P_{s}(x) P_{s}^{m}(x)= \begin{cases}2(-1)^{m / 2} \frac{s !(2 s) !}{(1+2)) !(s+m) !}, & \text { if } m \text { even } \\ 0, & \text { if } m \text { odd } .\end{cases}
$$

Finally we can perform the time integration. Using the explicit $\zeta$ mode function (4.11), one can analytically compute these integrals to find,

$$
\mathcal{I}_{1}=\frac{H^{2 s+5}}{m_{p l}^{6}(4 \epsilon)^{3}} \frac{1}{\left(k_{1} k_{2} k_{3}\right)^{3}}(1+s) \Gamma(s) \Gamma(2+s) \frac{k_{2}^{3}}{k_{1}^{s}\left(k_{2}+k_{3}\right)^{s+3}}\left(1+(s+3) \frac{k_{3}}{k_{2}}\right) .
$$

Putting the pieces together, we find for the non-Gaussianity,

$$
\begin{aligned}
& \left\langle\zeta\left(k_{1}, 0\right) \zeta\left(k_{2}, 0\right) \zeta\left(k_{3}, 0\right)\right\rangle \\
& \simeq \frac{1}{64 \pi} \lambda_{s} g_{s} \frac{H^{6}}{m_{p l}^{6} \epsilon^{3}}(1+s) \Gamma(s) \Gamma(2+s)\left(\frac{H}{\Lambda}\right)^{2 s-1} e^{-\pi \mu_{s}} e^{-\pi m_{\chi} / H} \\
& \quad \cdot \frac{\delta\left(\sum k_{i}\right)}{\left(k_{1} k_{2} k_{3}\right)^{3}} \frac{k_{3}^{s}}{\left(k_{2}+k_{3}\right)^{s}} \frac{k_{2}^{3} H^{3}}{\left(k_{2}+k_{3}\right)^{3}}\left(1+(s+1) \frac{k_{3}}{k_{2}}\right) \sum_{m=-s}^{s} c_{m} P_{s}^{m}\left(\hat{k}_{1} \cdot \hat{k}_{3}\right)+k_{2} \leftrightarrow k_{3}
\end{aligned}
$$

\footnotetext{
${ }^{11}$ Using the spherical harmonics addition theorem with $\phi_{1}=\phi_{2}=0$.
} 
which can be brought to a canonical form,

$$
\left\langle\zeta\left(k_{1}, 0\right) \zeta\left(k_{2}, 0\right) \zeta\left(k_{3}, 0\right)\right\rangle \simeq \mathcal{A}_{s+1 / 2} \frac{\Delta_{\zeta}(k)^{4}}{k^{6}} \mathcal{S}\left(k_{1}, k_{2}, k_{3}\right) \delta\left(\sum k_{i}\right) \sum_{m=-s}^{s} c_{m} P_{s}^{m}\left(\hat{k}_{1} \cdot \hat{k}_{3}\right)+k_{2} \leftrightarrow k_{3},
$$

where $\Delta_{\zeta}^{2}$ is the dimensionless primordial power spectrum, $\mathcal{S}\left(k_{1}, k_{2}, k_{3}\right)$ is a function of the ratios of $k_{i}$, and $\mathcal{A}$ is all remaining prefactors.

The angular dependence of the non-Gaussianity is given by,

$$
\left\langle\zeta\left(k_{1}, 0\right) \zeta\left(k_{2}, 0\right) \zeta\left(k_{3}, 0\right)\right\rangle \propto \sum_{m=-s}^{s} c_{m} P_{s}^{m}\left(\hat{k}_{1} \cdot \hat{k}_{3}\right)
$$

with the coefficients $c_{m}$ given by (4.52). The schematic form of the shape function $\mathcal{S}$ can be read off from (4.54), but the exact expression requires solving for the exact mode-functions of the higher spin particles in de Sitter space.

The corresponding non-Gaussianity parameter is given by,

$$
f_{N L} \simeq \lambda_{s} g_{s}(1+s) \Gamma(s) \Gamma(2+s)\left(\frac{H}{\Lambda}\right)^{2 s-1} e^{-\pi \mu_{s}} e^{-\pi m_{\chi} / H} \Delta_{\zeta}^{2},
$$

where the factor $\Gamma(s) \Gamma(2+s)$ is a relic of not having normalized the mode functions; we expect that as in the bosonic case, equation (A.77) of [13], the normalization of the exact solution of the mode functions scales with $1 / \Gamma(s)^{2}$, cancelling the $\Gamma(s)$ dependence of the 3-point function. The robust result is the scaling with the couplings $\lambda_{s}, g_{s}$, the ratio $H / \Lambda$, and the Boltzmann suppression due to both the higher-spin fermion and the spin- $1 / 2$ fermion, $e^{-\pi \mu_{s}} e^{-\pi \mu_{\chi}}$.

\subsection{The predictions of higher spin supersymmetry}

We can now read-off result for the three-point function $\langle\zeta \zeta \zeta\rangle$ given the higher spin supermultiplet. We simply add the contributions from the particle content of the half-integer superspin $\mathrm{Y}=s+1 / 2$ supermultiplet (3.1), given the non-Gaussianity from each spin derived in the previous section. The result is

$$
\begin{aligned}
\left\langle\zeta\left(k_{1}, 0\right) \zeta\left(k_{2}, 0\right) \zeta\left(k_{3}, 0\right)\right\rangle_{\mathrm{HS}-\mathrm{SUSY}}= & \left\langle\zeta\left(k_{1}, 0\right) \zeta\left(k_{2}, 0\right) \zeta\left(k_{3}, 0\right)\right\rangle_{s+1} \\
& +2 \times\left\langle\zeta\left(k_{1}, 0\right) \zeta\left(k_{2}, 0\right) \zeta\left(k_{3}, 0\right)\right\rangle_{s+1 / 2} \\
& +\left\langle\zeta\left(k_{1}, 0\right) \zeta\left(k_{2}, 0\right) \zeta\left(k_{3}, 0\right)\right\rangle_{s} \\
& \propto P_{s+1}\left(\hat{k}_{1} \cdot \hat{k}_{3}\right), \sum_{m=-s}^{s} P_{s}^{m}\left(\hat{k}_{1} \cdot \hat{k}_{3}\right), P_{s}\left(\hat{k}_{1} \cdot \hat{k}_{3}\right)
\end{aligned}
$$

where the last line indicates that the three terms in (4.58) have angular dependence given by $P_{s+1}, \sum_{m} P_{s}^{m}$, and $P_{s}$ respectively. The relative amplitudes are determined by the mass spectrum of the theory.

The quantitative amplitude of this signal is, as in the non-supersymmetric bosonic case [13], generally small $f_{N L} \lesssim \mathcal{O}(1)$. The primary obstruction making $f_{N L}$ any larger 
than this is perturbativity of the interaction strength, which at the very least, requires $\lambda_{s}(H / \Lambda)^{s-1} \ll 1$ and $g_{s}(H / \Lambda)^{s} \ll 1$, as these are the effective interaction strengths, e.g. appearing in (4.41). Non-Gaussianity of this size is not what would traditionally be referred to as 'large', but it can be considerably larger than the slow-roll suppressed single-field slow-roll value, and is within reach for CMB-S4 [84].

The analysis can be repeated for the case of embedding the higher spin particles inside the integer superspin supermultiplet (3.3) instead of the half integer one we have used as an example. In that case the particle contained is $(s+1 / 2, s, s, s-1 / 2)$ therefore one can immediately read off the result. The known $P_{s}(\cos \theta)$ dependence of spin- $s$ bosons $[12,13]$ is accompanied by two towers of associated Legendre polynomials, $\sum_{m} P_{s}^{m}$ and $\sum_{m} P_{s-1}^{m}$, from the $s+1 / 2$ and $s-1 / 2$ fermions respectively.

\section{Discussion}

Precision measurements of the cosmic microwave background provide an unprecedented opportunity to search for new physics in the early universe. The 3-point function of primordial curvature perturbations, $\langle\zeta \zeta \zeta\rangle$, colloquially referred to as the non-Gaussianity, is sensitive to any new degrees of freedom, including those that are naively too heavy to be excited. One of the most striking results of this research program is the non-Gaussianity due to higher spin particles, and in particular the angular dependence $\langle\zeta \zeta \zeta\rangle \propto P_{s}(\cos \theta)$ due to the exchange of a single spin- $s$ boson [12]. This prompted a flurry of activity, and possibilities for observing [87-95] the signature of higher spin particles.

Higher spin fermions have heretofore been left out of this discussion, but insofar as higher spin theory is understood as a limit of quantum gravity, namely superstring theory, fermions are built into the theory. This is required by the supersymmetric nature of the theory, which is itself a powerful tool for the incorporation of fermions into string theory, e.g. the construction of fermionic D-brane actions is accomplished by relying on the underlying supersymmetry of the theory [96-99]. Guided by this, and building on recent developments in the construction of supersymmetric higher spin theories [23-32, 34-37], we have studied the imprint of higher spin supersymmetry at the cosmological collider.

The main result of this paper is a characteristic pattern of the angular dependence of $\langle\zeta \zeta \zeta\rangle$ due to the exchange of higher spin superpartners. We find the $P_{s}(\cos \theta)$ signature of higher spin boson exchange, with $\theta$ the angle between the short and long wavelength modes, comes along with a $P_{s+1}(\cos \theta)$ and a tower of associated Legendre polynomials, arising from a spin- $s+1$ boson and a pair of spin- $s+1 / 2$ fermions. For a variant description of higher spin supermultiplet, the partner contributions can be instead two towers of associated Legendre polynomials. The amplitude of the signal is generically not large by comparison to other known sources (e.g. [100, 101]), as already known for the non-supersymmetric bosonic case [13], so it is indeed the angular dependence which gives this signal its elevated status. Given this, in this work we have not endeavored to do a rigorous and precise calculation of the shape-function, which requires explicitly solving the mode-functions and computing involved integrals [13]. This latter difficulty motivated the development of the cosmological bootstrap [102], which might be a promising direction to take this work as well. 
Remarkably, we have been able to derive these results despite not having a complete theory or model realization of higher spin supergravity inflation. Progress despite incomplete knowledge is a familiar situation in theoretical physics, for example, supersymmetry and the Green-Schwarz mechanism, all work to date pertaining to M-theory [103, 104], or in a more recent context, Double Field Theory [105]. To overcome this, we have constructed an effective theory that combines higher spin supersymmetry with de Sitter supergravity and the effective field theory of inflation, to describe a higher spin sector minimally coupled to the inflationary sector such that the higher spin sector retains on-shell supersymmetry. This allowed us to use supersymmetry considerations to deduce the field content and interactions of the higher spin fields with the curvature perturbation.

There are a number of ways forward from here. We have not considered yet the interactions with the graviton, or for that the matter, the gravitino. The former of these, corresponding to primordial gravitational waves $\gamma$, itself can lead to an interesting three point function, $\langle\gamma \zeta \zeta\rangle$, probed by cross-correlation with CMB B-mode polarization [106]. Starting from an effective field theory guided guess for the relevant interaction,

$$
\mathcal{L}_{\gamma H S}=\frac{\hat{\lambda}_{s}}{\Lambda^{s-2}} \partial_{i_{1} \ldots i_{s-2}} \dot{\gamma}_{i_{s-1} i_{s}} \bar{\chi} \psi^{i_{1} \ldots i_{s}}+\text { h.c. }
$$

the $\langle\gamma \zeta \zeta\rangle$ computed can be straight-forwardly worked out in a fashion similar to section 4 . We provide the calculations for this in appendix B, and here we give the result:

$$
\begin{aligned}
& \lim _{k_{1} \ll k_{2}, k_{3}}\left\langle\gamma^{\lambda}\left(k_{1}\right) \zeta\left(k_{2}\right) \zeta\left(k_{3}\right)\right\rangle=\hat{\lambda}_{s} g_{s}\left(\frac{H}{\Lambda}\right)^{2 s-2} \frac{H^{6}}{m_{p l}^{6}} \frac{2 \sqrt{2}}{(4 \epsilon)^{2}} e^{-\pi m_{\chi} / H} e^{-\pi \mu_{s}} \delta\left(\sum k_{i}\right) \\
& \cdot \mathcal{S}\left(k_{1}, k_{2}, k_{3}\right) \int \frac{d \Omega}{(2 \pi)^{3}} P_{s-2}\left(\hat{k}_{1} \cdot \hat{q}\right) \varepsilon_{i j}^{\lambda}\left(\hat{k}_{1}\right) \sum_{\lambda^{\prime}= \pm 2} \varepsilon^{\lambda^{\prime} i j}(\hat{q}) \hat{P}_{s}^{\lambda^{\prime}}\left(\hat{k}_{3} \cdot \hat{q}\right) \mathcal{E}_{2}^{\lambda^{\prime}}\left(\hat{k}_{3} \cdot \hat{q}\right)+k_{2} \leftrightarrow k_{3},
\end{aligned}
$$

where $\lambda= \pm 2$ is the helicity of the external graviton, $\epsilon_{i j}$ is the spin-2 polarization tensor, $\hat{P}_{s}^{\lambda}(x) \equiv\left(1-x^{2}\right)^{-\lambda / 2} P_{s}^{\lambda}(x)$ and $\mathcal{E}_{2}^{\lambda}\left(\hat{k}_{1} \cdot \hat{k}_{3}\right)=\epsilon_{i j}^{\lambda}\left(\hat{k}_{1}\right) \hat{k}_{3}^{i} \hat{k}_{3}^{j}$ as in [13], and we have put all $k$-dependence in the function $\mathcal{S}$. This result is characterized by an angular dependence that is an integral over Legendre and associated Legendre polynomials,

$$
\left\langle\gamma^{\lambda} \zeta \zeta\right\rangle \propto \sum_{\lambda^{\prime}= \pm 2} \int d \cos \theta_{q} P_{s-2}\left(\hat{k}_{1} \cdot \hat{q}\right) \varepsilon_{i j}^{\lambda}\left(\hat{k}_{1}\right) \varepsilon^{\lambda^{\prime} i j}(\hat{q}) \hat{P}_{s}^{\lambda^{\prime}}\left(\hat{k}_{3} \cdot \hat{q}\right) \mathcal{E}_{2}^{\lambda^{\prime}}\left(\hat{k}_{3} \cdot \hat{q}\right)
$$

where $\theta_{q}$ is the angle $\hat{q}$ makes in the plane. In the supersymmetric context, this would be joined with contributions from additional interactions. This requires careful consideration of the gravity multiplet, as is the focus of the supersymmetric EFT of inflation [42]. We postpone this analysis to future work.

On the theoretical front, an important next step is to construct the full theory of spontaneously broken supersymmetry (as in de Sitter supergravity and the supersymmetric EFT of inflation) and interacting higher spin fields. From this one can generalize the analysis here to situations where the higher spin fields themselves contribute to the supersymmetry breaking, or perhaps even drive inflation. We leave this possibility, and a host of observational implications, to future work. 
As a concluding remark, we would like to express and share our enthusiasm for the work of scientists who are searching for signals of supersymmetry in the cosmos, a sentiment expressed by one of the authors in [107]. As argued for in this work, the non-Gaussianity of the CMB may prove to be a powerful tool of discovery, and with some good fortune, perhaps more and different such tools will later emerge for the SUSY search at the Cosmic Collider.

"I wantmy@!\%!**\#“\# signal...where is it? It's time to pay the piper!!"

- B. Richter, in conversation overheard between the SLAC Director and physicist B.J. Bjorken.

\section{Acknowledgments}

This paper is dedicated to the memory of Burton Richter. The authors thank Hayden Lee for many helpful and insightful discussions during the completion of this work. The research of S.J.G. and K.K. is supported by the endowment of the Ford Foundation Professorship of Physics at Brown University. This work was partially supported by the U.S. National Science Foundation grant PHY-1315155.

\section{A Spin-s polarization vectors}

This appendix discusses some relevant preliminaries and definitions relating to the free theory of higher spin fields in de Sitter space, which can also be found in [13]. Following [13], vectors will be denoted here in boldface, e.g. $\mathbf{k}$.

It is convenient to project the spinning field, $\sigma_{\mu_{1} \ldots \mu_{s}}$ onto spatial slices, which we can then write as

$$
\sigma_{i_{1} \ldots i_{n} \eta \ldots \eta}=\sum_{\lambda} \sigma_{n, s}^{\lambda} \varepsilon_{i_{1} \ldots i_{n}}^{\lambda}
$$

Here, the $\mathrm{s}$ index refers to the spin, $\mathrm{n}$ refers to the 'spatial spin,' and $\lambda$ is the helicity of the field. $\varepsilon_{i_{s} \ldots i_{n}}^{\lambda}$ is a normalized, totally symmetric tensor with spin s and helicity $\lambda$. It must satisfy:

$$
\varepsilon_{i_{1} \ldots i_{s}}^{\lambda}=\varepsilon_{\left(i_{1} \ldots i_{s}\right)}^{\lambda}, \quad \varepsilon_{i i i_{3} \ldots i_{s}}^{\lambda}=0, \quad \hat{k}_{i_{1}} \ldots \hat{k}_{i_{r}} \varepsilon_{i_{1} \ldots i_{s}}^{\lambda}=0 \quad \text { for } r>s-|\lambda|,
$$

corresponding to the symmetric, traceless, and transverse properties. These properties of the polarization tensor imply that we can decompose it into transverse and longitudinal parts as,

$$
\varepsilon_{i_{1} \ldots i_{s}}^{\lambda}(\hat{\mathbf{k}}, \varepsilon)=\varepsilon_{\left(i_{1} \ldots i_{\lambda}\right.}^{\lambda}(\varepsilon) f_{\left.i_{\lambda+1} \ldots i_{s}\right)}(\hat{\mathbf{k}}),
$$

where $\varepsilon_{i_{1} \ldots i_{\lambda}}^{\lambda}$ is a maximally transverse polarization tensor, constructed out of polarization vectors $\varepsilon^{ \pm}$that are perpendicular to $\hat{\mathbf{k}}$. We must have that $\varepsilon^{+}=\left(\varepsilon^{-}\right) *$, so that $\varepsilon_{i_{1} \ldots i_{\lambda}}^{\lambda}$ can be specified, up to a phase, by a single polarization vector $\varepsilon$. We have also defined $f_{i_{\lambda+1} \ldots i_{s}}$ as the longitudinal part of the associated Legendre polynomial, after contraction with momenta. 
We then define,

$$
F_{s}^{\lambda}=q_{i_{1}} \ldots q_{i_{s}} \varepsilon_{i_{1} \ldots i_{s}}^{\lambda}(\mathbf{k}) .
$$

The symmetry properties of $\varepsilon$ imply that $F_{s}^{\lambda}$ takes the form [13], in $\mathrm{d}=3$ spatial dimensions,

$$
F_{s}^{\lambda} \propto z \hat{P}_{s}^{\lambda}
$$

where $z \equiv q_{i_{1}} \ldots q_{i_{\lambda}} \varepsilon_{i_{1} \ldots i_{\lambda}}^{\lambda}$, and $\hat{P}$ defined via

$$
P_{s}^{\lambda}(\theta, \phi)=\sin ^{\lambda} \theta \hat{P}_{s}^{\lambda}(\theta, \phi),
$$

where $P_{s}^{\lambda}$ is the associated Legendre polynomial. For the special case of $\lambda=0$, which appears in the calculation of 3-point functions after enforcing momentum conservation, the othonormality of differing helicity states $\lambda$ and $\lambda^{\prime}$, and the transverse property (A.2), one has

$$
q_{i_{1}} \ldots q_{i_{s}} \varepsilon_{i_{1} \ldots i_{s}}^{0}(\hat{\mathbf{k}}) \propto P_{s}(\hat{\mathbf{q}} \cdot \hat{\mathbf{k}}),
$$

with magnitude $\left|q_{1}\right|^{s}$, leading to the characteristic angular dependence of the three-point function for spin- $s$ boson exchange. Moreover, the transverse property also implies that the only $\sigma_{n, s}^{0}$ that enters the correlation function is $n=s$.

\section{B Details of $\langle\gamma \zeta \zeta\rangle$ calculation}

In this appendix, we further explicate the derivation of the tensor-scalar-scalar correlation function. We will limit our analysis to the single-exchange diagram shown in figure 2 .

This diagram has the same form as figure 1a, however now we have an external graviton carrying momentum $k_{1}$ instead of $\zeta$. The relevant interaction Lagrangian we will consider is

$$
\mathcal{L}=\frac{\hat{\lambda}_{s}}{\Lambda^{s-2}} \partial_{i_{1} \ldots i_{s-2}} \dot{\gamma}_{i_{s-1} i_{s}} \bar{\chi} \psi^{i_{1} \ldots i_{s}}+\frac{g_{s}}{\Lambda^{s}} \dot{\zeta} \partial_{i_{1} \ldots i_{s}} \zeta \bar{\chi} \psi^{i_{1} \ldots i_{s}}+h . c .
$$

which corresponds to two interaction Hamiltonians

$$
\begin{aligned}
H_{\text {int }_{1}} & =\frac{\hat{\lambda}_{s}}{\Lambda^{s-2}} \int d^{3} x \frac{1}{a^{2 s-2}} \partial_{i_{1} \ldots i_{s-2}} \gamma_{i_{s-1} i_{s}}^{\prime} \bar{\chi} \psi^{i_{1} \ldots i_{s}}+h . c . \\
H_{\text {int }_{2}} & =\frac{g_{s}}{\Lambda^{s}} \int d^{3} x \frac{1}{a^{2 s-2}} \zeta^{\prime} \partial_{i_{1} \ldots i_{s}} \zeta \bar{\chi} \psi^{i_{1} \ldots i_{s}}+h . c . .
\end{aligned}
$$

As in the $\langle\zeta \zeta \zeta\rangle$ calculation, we would like to expand in Fourier modes. The graviton can be expanded in helicity modes as given in [13]:

$$
\gamma_{i j}(\mathbf{k}, \eta)=\sum_{\lambda= \pm 2} \varepsilon_{i j}^{\lambda}(\mathbf{k}) \gamma_{k}^{\lambda}(\eta) b(\mathbf{k}, \lambda)+h . c .,
$$

where the graviton mode function, $\gamma_{k}^{\lambda}$, is given by

$$
\gamma_{k}^{\lambda}(\eta)=\frac{\sqrt{2} H}{m_{p l}} \frac{1}{\sqrt{2 k^{3}}}(1+i k \eta) e^{-i k \eta} .
$$




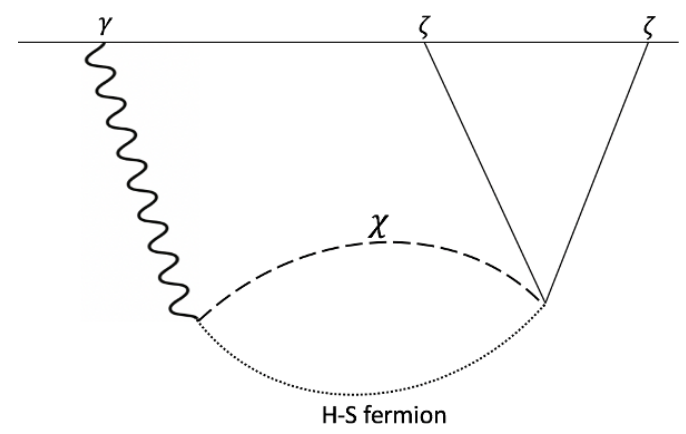

Figure 2. Diagram contributing to $\langle\gamma \zeta \zeta\rangle$.

With the mode functions in hand we can compute the tensor-scalar-scalar three point function $\langle\gamma \zeta \zeta\rangle$. As before, we would like to expand each interaction Hamiltonian in momentum space and compute the correlator. Much of the calculation remains the same as in the $\langle\zeta \zeta \zeta\rangle$ case, however, there are some subtleties. The angular dependence due to $H_{\text {int }_{2}}$ remains largely the same, however now due to the fact that $\lambda \neq 0$, we have $H_{\text {int }_{2}} \propto \mathcal{E}_{2}^{\lambda}\left(\hat{k}_{3} \cdot \hat{q}\right) \hat{P}_{s}^{\lambda}\left(\hat{k}_{3} \cdot \hat{q}\right)$, rather than simply $P_{s}\left(\hat{k}_{3} \cdot \hat{q}\right)$ as in the $\langle\zeta \zeta \zeta\rangle$ case. This arises from the definition (A.5), and we have defined $\mathcal{E}_{2}^{\lambda}\left(\hat{k}_{3} \cdot \hat{q}\right)=\varepsilon_{i j}^{\lambda}\left(\hat{k}_{3}\right) \hat{q}^{i} \hat{q}^{j}$. The angular dependence of $H_{\text {int }_{1}}$ is similarly complicated due to the contraction with $\gamma_{i_{s-1}, i_{s}}$.

After expanding in momentum space and following a similar procedure as in the $\langle\zeta \zeta \zeta\rangle$ calculation, we obtain

$$
\begin{aligned}
\left\langle\gamma^{\lambda} \zeta \zeta\right\rangle= & 8 \frac{\hat{\lambda}_{s} g_{s}}{\Lambda^{2 s-2}} \operatorname{Re} \gamma_{k_{1}}^{\lambda}(0) \zeta_{k_{2}}(0) \zeta_{k_{3}}(0) \int \frac{d \eta^{\prime}}{a\left(\eta^{\prime}\right)^{2 s-3}} \int \frac{d \eta^{\prime \prime}}{a\left(\eta^{\prime \prime}\right)^{2 s-3}} \int \frac{d^{3} \ell}{(2 \pi)^{3}} \\
& \cdot \gamma_{k_{1}}^{\lambda *^{\prime}}\left(\eta^{\prime}\right) \zeta_{k_{2}}^{*^{\prime}}\left(\eta^{\prime \prime}\right) \zeta_{k_{3}}^{*}\left(\eta^{\prime \prime}\right) \bar{\chi}_{\ell}\left(\eta^{\prime}\right) \chi_{\ell}\left(\eta^{\prime \prime}\right) \sum_{\lambda^{\prime}= \pm 2} \psi_{s, s, \ell+k_{1}}^{\lambda^{\prime}}\left(\eta^{\prime}\right) \bar{\psi}_{s, s, \ell+k_{1}}^{\lambda^{\prime}}\left(\eta^{\prime \prime}\right) \\
& \cdot\left|k_{1}\right|^{s-2}\left|k_{3}\right|^{s} P_{s-2}\left(\hat{k}_{1} \cdot \hat{q}\right) \varepsilon_{i j}^{\lambda}\left(\hat{k}_{1}\right) \varepsilon^{\lambda^{\prime} i j}(\hat{q}) \hat{P}_{s}^{\lambda^{\prime}}\left(\hat{k}_{3} \cdot \hat{q}\right) \mathcal{E}_{2}^{\lambda^{\prime}}\left(\hat{k}_{3} \cdot \hat{q}\right) \delta\left(\sum k_{i}\right),
\end{aligned}
$$

where we have defined $\mathcal{E}$ as in $[13]$ as $\mathcal{E}_{2}^{\lambda}\left(\hat{k}_{1} \cdot \hat{k}_{3}\right)=\epsilon_{i j}^{\lambda}\left(\hat{k}_{1}\right) \hat{k}_{3}^{i} \hat{k}_{3}^{j}$, and where $\vec{q} \equiv \vec{\ell}+\overrightarrow{k_{1}}$. The mode functions for $\chi$ remain the same as in the previous calculation, given by (4.22), and we approximate that $\psi_{s, s}^{ \pm 2}$ by their super-horizon scaling, which is the same as (4.31). Plugging in the explicit expressions for the mode functions and substituting $a(\eta)=-\frac{1}{H \eta}$, we have,

$$
\begin{aligned}
\left\langle\gamma^{\lambda} \zeta \zeta\right\rangle= & \frac{\hat{\lambda}_{s} g_{s}}{\Lambda^{2 s-2}} H^{2 s} \frac{H^{6}}{m_{p l}^{6}} \frac{2 \sqrt{2}}{(4 \epsilon)^{2}} \frac{1}{k_{1}^{3} k_{2}^{3} k_{3}^{3}} e^{-\pi m_{\chi} / H} e^{-\pi \mu_{s}}\left|k_{1}\right|^{s-2}\left|k_{3}\right|^{s} \delta\left(\sum k_{i}\right) \\
& \cdot \operatorname{Re} \int d \eta^{\prime} \eta^{\prime s}\left(k_{1}^{2} \eta^{\prime}\right) e^{i k_{1} \eta^{\prime}} \int d \eta^{\prime \prime} \eta^{\prime \prime s}\left(k_{2}^{2} \eta^{\prime \prime}\right)\left(1+i k_{3} \eta^{\prime \prime}\right) e^{-i\left(k_{2}+k_{3}\right) \eta^{\prime \prime}} \\
& \int \frac{d^{3} \ell}{(2 \pi)^{3}} P_{s-2}\left(\hat{k}_{1} \cdot \hat{q}\right) \varepsilon_{i j}^{\lambda}\left(\hat{k}_{1}\right) \sum_{\lambda^{\prime}= \pm 2} \varepsilon^{\lambda^{\prime} i j}(\hat{q}) \hat{P}_{s}^{\lambda^{\prime}}\left(\hat{k}_{3} \cdot \hat{q}\right) \mathcal{E}_{2}^{\lambda^{\prime}}\left(\hat{k}_{3} \cdot \hat{q}\right)+k_{2} \leftrightarrow k_{3} .
\end{aligned}
$$

where $\hat{P}_{s}^{\lambda}(x) \equiv\left(1-x^{2}\right)^{-\lambda / 2} P_{s}^{\lambda}(x)$ as in [13].

For ease of notation, let us denote this as,

$$
\left\langle\gamma^{\lambda} \zeta \zeta\right\rangle=\mathcal{J}_{1} \mathcal{J}_{2} \mathcal{J}_{3}
$$


where $\mathcal{J}_{1}$ is the prefactor, $\mathcal{J}_{2}$ are the time integrals and $\mathcal{J}_{3}$ is the momentum integral. Performing the time integration and keeping only the real part yields

$$
\mathcal{J}_{2}=\frac{k_{1}^{-s} k_{2}^{2}\left(k_{2}+k_{3}(s+3)\right) \Gamma(s+2)^{2}}{\left(k_{2}+k_{3}\right)^{s+3}} .
$$

In $\mathcal{J}_{3}$ we can perform the integration over $\ell$, enforcing a cutoff at $H$, leaving only the angular integral. Putting everything together, we obtain,

$$
\begin{aligned}
\lim _{k_{1} \ll k_{2}, k_{3}}\left\langle\gamma^{\lambda}\left(k_{1}\right) \zeta\left(k_{2}\right) \zeta\left(k_{3}\right)\right\rangle= & \frac{\hat{\lambda}_{s} g_{s}}{\Lambda^{2 s-2}} \frac{H^{2 s+6}}{m_{p l}^{6}} \frac{2 \sqrt{2}}{(4 \epsilon)^{2}} e^{-\pi m_{\chi} / H} e^{-\pi \mu_{s}} \delta\left(\sum k_{i}\right) \\
& \cdot \frac{H^{3} k_{1}^{-2} k_{2}^{3} k_{3}^{s}}{\left(k_{1} k_{2} k_{3}\right)^{3}\left(k_{2}+k_{3}\right)^{s+3}}\left(1+\frac{k_{3}}{k_{2}}(s+3)\right) \Gamma(s+2)^{2} \\
& \cdot \int \frac{d \Omega}{(2 \pi)^{3}} P_{s-2}\left(\hat{k}_{1} \cdot \hat{q}\right) \varepsilon_{i j}^{\lambda}\left(\hat{k}_{1}\right) \varepsilon^{\lambda i j}(\hat{q}) \hat{P}_{s}^{\lambda}\left(\hat{k}_{3} \cdot \hat{q}\right) \mathcal{E}_{2}^{\lambda}\left(\hat{k}_{3} \cdot \hat{q}\right)+k_{2} \leftrightarrow k_{3} .
\end{aligned}
$$

In a canonical form we can write this as,

$$
\begin{aligned}
& \lim _{k_{1} \ll k_{2}, k_{3}}\left\langle\gamma^{\lambda}\left(k_{1}\right) \zeta\left(k_{2}\right) \zeta\left(k_{3}\right)\right\rangle=\hat{\lambda}_{s} g_{s}\left(\frac{H}{\Lambda}\right)^{2 s-2} \frac{H^{6}}{m_{p l}^{6}} \frac{2 \sqrt{2}}{(4 \epsilon)^{2}} e^{-\pi m_{\chi} / H} e^{-\pi \mu_{s}} \delta\left(\sum k_{i}\right) \\
& \cdot \mathcal{S}\left(k_{1}, k_{2}, k_{3}\right) \sum_{\lambda^{\prime}= \pm 2} \int \frac{d \Omega}{(2 \pi)^{3}} P_{s-2}\left(\hat{k}_{1} \cdot \hat{q}\right) \varepsilon_{i j}^{\lambda}\left(\hat{k}_{1}\right) \varepsilon^{\lambda^{\prime} i j}(\hat{q}) \hat{P}_{s}^{\lambda^{\prime}}\left(\hat{k}_{3} \cdot \hat{q}\right) \mathcal{E}_{2}^{\lambda^{\prime}}\left(\hat{k}_{3} \cdot \hat{q}\right)+k_{2} \leftrightarrow k_{3} .
\end{aligned}
$$

The angular dependence is given by

$$
\left\langle\gamma^{\lambda} \zeta \zeta\right\rangle \propto \sum_{\lambda^{\prime}= \pm 2} \int d \cos \theta_{q} P_{s-2}\left(\hat{k}_{1} \cdot \hat{q}\right) \varepsilon_{i j}^{\lambda}\left(\hat{k}_{1}\right) \varepsilon^{\lambda^{\prime} i j}(\hat{q}) \hat{P}_{s}^{\lambda^{\prime}}\left(\hat{k}_{3} \cdot \hat{q}\right) \mathcal{E}_{2}^{\lambda^{\prime}}\left(\hat{k}_{3} \cdot \hat{q}\right)
$$

where $\theta_{q}$ is the angle $\hat{q}$ makes in the plane.

Open Access. This article is distributed under the terms of the Creative Commons Attribution License (CC-BY 4.0), which permits any use, distribution and reproduction in any medium, provided the original author(s) and source are credited.

\section{References}

[1] Planck collaboration, Planck 2018 results. I. Overview and the cosmological legacy of Planck, arXiv:1807.06205 [INSPIRE].

[2] D. Wands, K.A. Malik, D.H. Lyth and A.R. Liddle, A New approach to the evolution of cosmological perturbations on large scales, Phys. Rev. D 62 (2000) 043527 [astro-ph/0003278] [INSPIRE].

[3] S. Weinberg, Adiabatic modes in cosmology, Phys. Rev. D 67 (2003) 123504 [astro-ph/0302326] [INSPIRE].

[4] G.I. Rigopoulos and E.P.S. Shellard, The separate universe approach and the evolution of nonlinear superhorizon cosmological perturbations, Phys. Rev. D 68 (2003) 123518 [astro-ph/0306620] [INSPIRE]. 
[5] D.H. Lyth, K.A. Malik and M. Sasaki, A General proof of the conservation of the curvature perturbation, JCAP 05 (2005) 004 [astro-ph/0411220] [INSPIRE].

[6] D. Langlois and F. Vernizzi, Conserved non-linear quantities in cosmology, Phys. Rev. D 72 (2005) 103501 [astro-ph/0509078] [inSPIRE].

[7] V. Assassi, D. Baumann and D. Green, Symmetries and Loops in Inflation, JHEP 02 (2013) 151 [arXiv: 1210.7792] [inSPIRE].

[8] L. Senatore and M. Zaldarriaga, The constancy of $\zeta$ in single-clock Inflation at all loops, JHEP 09 (2013) 148 [arXiv:1210.6048] [INSPIRE].

[9] X. Chen and Y. Wang, Quasi-Single Field Inflation and Non-Gaussianities, JCAP 04 (2010) 027 [arXiv: 0911.3380] [INSPIRE].

[10] X. Chen and Y. Wang, Large non-Gaussianities with Intermediate Shapes from Quasi-Single Field Inflation, Phys. Rev. D 81 (2010) 063511 [arXiv:0909.0496] [InSPIRE].

[11] X. Chen, M.H. Namjoo and Y. Wang, Quantum Primordial Standard Clocks, JCAP 02 (2016) 013 [arXiv: 1509.03930] [inSPIRE].

[12] N. Arkani-Hamed and J. Maldacena, Cosmological Collider Physics, arXiv:1503.08043 [INSPIRE].

[13] H. Lee, D. Baumann and G.L. Pimentel, Non-Gaussianity as a Particle Detector, JHEP 12 (2016) 040 [arXiv: 1607.03735] [inSPIRE].

[14] E. Majorana, Relativistic theory of particles with arbitrary intrinsic angular momentum, Nuovo Cim. 9 (1932) 335 [inSPIRE].

[15] T. Noumi, T. Takeuchi and S. Zhou, String Regge trajectory on de Sitter space and implications to inflation, arXiv:1907.02535 [INSPIRE].

[16] D. Lüst and E. Palti, A Note on String Excitations and the Higuchi Bound, arXiv:1907.04161 [INSPIRE].

[17] D.J. Gross, Strings at superPlanckian energies: In search of the string symmetry, Phil. Trans. Roy. Soc. Lond. A 329 (1989) 401 [inSPIRE].

[18] E. Witten, The search for higher symmetry in string theory, Phil. Trans. Roy. Soc. Lond. A 329 (1989) 349 [inSPIRE].

[19] D.J. Gross and P.F. Mende, String Theory Beyond the Planck Scale, Nucl. Phys. B 303 (1988) 407 [INSPIRE].

[20] E.S. Fradkin and M.A. Vasiliev, Candidate to the Role of Higher Spin Symmetry, Annals Phys. 177 (1987) 63 [INSPIRE].

[21] R. Haag, J.T. Lopuszanski and M. Sohnius, All Possible Generators of Supersymmetries of the s Matrix, Nucl. Phys. B 88 (1975) 257 [INSPIRE].

[22] I.L. Buchbinder, S.J. Gates Jr. and K. Koutrolikos, Superfield continuous spin equations of motion, Phys. Lett. B 793 (2019) 445 [arXiv:1903.08631] [INSPIRE].

[23] S.M. Kuzenko, A.G. Sibiryakov and V.V. Postnikov, Massless gauge superfields of higher half integer superspins, JETP Lett. 57 (1993) 534 [INSPIRE].

[24] S.M. Kuzenko and A.G. Sibiryakov, Massless gauge superfields of higher integer superspins, JETP Lett. 57 (1993) 539 [INSPIRE]. 
[25] S.M. Kuzenko and A.G. Sibiryakov, Free massless higher superspin superfields on the anti-de Sitter superspace, Phys. Atom. Nucl. 57 (1994) 1257 [arXiv:1112.4612] [InSPIRE].

[26] I.L. Buchbinder, S.M. Kuzenko and A.G. Sibiryakov, Quantization of higher spin superfields in the anti-de Sitter superspace, Phys. Lett. B 352 (1995) 29 [hep-th/9502148] [INSPIRE].

[27] S.J. Gates Jr., S.M. Kuzenko and A.G. Sibiryakov, $N=2$ supersymmetry of higher superspin massless theories, Phys. Lett. B 412 (1997) 59 [hep-th/9609141] [INSPIRE].

[28] S.J. Gates Jr., S.M. Kuzenko and A.G. Sibiryakov, Towards a unified theory of massless superfields of all superspins, Phys. Lett. B 394 (1997) 343 [hep-th/9611193] [INSPIRE].

[29] S.J. Gates Jr. and S.M. Kuzenko, $4 D, N=1$ higher spin gauge superfields and quantized twistors, JHEP 10 (2005) 008 [hep-th/0506255] [INSPIRE].

[30] S.J. Gates Jr. and K. Koutrolikos, On $4 D, \mathcal{N}=1$ massless gauge superfields of arbitrary superhelicity, JHEP 06 (2014) 098 [arXiv: 1310.7385] [INSPIRE].

[31] I.L. Buchbinder and K. Koutrolikos, BRST Analysis of the Supersymmetric Higher Spin Field Models, JHEP 12 (2015) 106 [arXiv:1510.06569] [INSPIRE].

[32] S.J. Gates Jr. and K. Koutrolikos, From Diophantus to Supergravity and massless higher spin multiplets, JHEP 11 (2017) 063 [arXiv:1707.00194] [INSPIRE].

[33] D. Sorokin and M. Tsulaia, Supersymmetric Reducible Higher-Spin Multiplets in Various Dimensions, Nucl. Phys. B 929 (2018) 216 [arXiv:1801.04615] [InSPIRE].

[34] Y.M. Zinoviev, Massive $N=1$ supermultiplets with arbitrary superspins, Nucl. Phys. B $\mathbf{7 8 5}$ (2007) 98 [arXiv:0704.1535] [inSPIRE].

[35] I.L. Buchbinder, M.V. Khabarov, T.V. Snegirev and Y.M. Zinoviev, Lagrangian formulation of the massive higher spin $N=1$ supermultiplets in AdS $S_{4}$ space, Nucl. Phys. B 942 (2019) 1 [arXiv: 1901.09637 ] [INSPIRE].

[36] S.J. Gates Jr., S.M. Kuzenko and G. Tartaglino-Mazzucchelli, New massive supergravity multiplets, JHEP 02 (2007) 052 [hep-th/0610333] [INSPIRE].

[37] S.J. Gates Jr. and K. Koutrolikos, A dynamical theory for linearized massive superspin 3/2, JHEP 03 (2014) 030 [arXiv: 1310.7387] [INSPIRE].

[38] C. Cheung, P. Creminelli, A.L. Fitzpatrick, J. Kaplan and L. Senatore, The Effective Field Theory of Inflation, JHEP 03 (2008) 014 [arXiv:0709.0293] [INSPIRE].

[39] E.A. Bergshoeff, D.Z. Freedman, R. Kallosh and A. Van Proeyen, Pure de Sitter Supergravity, Phys. Rev. D 92 (2015) 085040 [Erratum ibid. D 93 (2016) 069901] [arXiv: 1507.08264] [INSPIRE].

[40] R. Kallosh and T. Wrase, de Sitter Supergravity Model Building, Phys. Rev. D 92 (2015) 105010 [arXiv: 1509.02137] [INSPIRE].

[41] R. Kallosh, Matter-coupled de Sitter Supergravity, Theor. Math. Phys. 187 (2016) 695 [arXiv: 1509. 02136] [INSPIRE].

[42] L.V. Delacretaz, V. Gorbenko and L. Senatore, The Supersymmetric Effective Field Theory of Inflation, JHEP 03 (2017) 063 [arXiv:1610.04227] [INSPIRE].

[43] S.J. Gates Jr., M.T. Grisaru and S. Penati, Holomorphy, minimal homotopy and the 4-D, $N=1$ supersymmetric Bardeen-Gross-Jackiw anomaly, Phys. Lett. B 481 (2000) 397 [hep-th/0002045] [INSPIRE]. 
[44] S.J. Gates Jr., M.T. Grisaru, M.E. Knutt, S. Penati and H. Suzuki, Supersymmetric gauge anomaly with general homotopic paths, Nucl. Phys. B 596 (2001) 315 [hep-th/0009192] [INSPIRE].

[45] S.J. Gates Jr., M.T. Grisaru, M.E. Knutt and S. Penati, The Superspace WZNW action for 4-D, $N=1$ supersymmetric QCD, Phys. Lett. B 503 (2001) 349 [hep-ph/0012301] [INSPIRE].

[46] M.B. Green and J.H. Schwarz, Anomaly Cancellation in Supersymmetric D $=10$ Gauge Theory and Superstring Theory, Phys. Lett. 149B (1984) 117 [INSPIRE].

[47] E.A. Bergshoeff and M. de Roo, The Quartic Effective Action of the Heterotic String and Supersymmetry, Nucl. Phys. B 328 (1989) 439 [INSPIRE].

[48] C. Vafa and E. Witten, A One loop test of string duality, Nucl. Phys. B 447 (1995) 261 [hep-th/9505053] [INSPIRE].

[49] M.J. Duff, J.T. Liu and R. Minasian, Eleven-dimensional origin of string-string duality: A One loop test, Nucl. Phys. B 452 (1995) 261 [hep-th/9506126] [INSPIRE].

[50] E. Witten, Five-brane effective action in M-theory, J. Geom. Phys. 22 (1997) 103 [hep-th/9610234] [INSPIRE].

[51] K. Peeters, P. Vanhove and A. Westerberg, Supersymmetric higher derivative actions in ten-dimensions and eleven-dimensions, the associated superalgebras and their formulation in superspace, Class. Quant. Grav. 18 (2001) 843 [hep-th/0010167] [INSPIRE].

[52] M.B. Green and M. Gutperle, Effects of D instantons, Nucl. Phys. B 498 (1997) 195 [hep-th/9701093] [INSPIRE].

[53] J.M. Maldacena, The Large $N$ limit of superconformal field theories and supergravity, Int. J. Theor. Phys. 38 (1999) 1113 [hep-th/9711200] [InSPIRE].

[54] D. Baumann and L. McAllister, Inflation and String Theory, Cambridge Monographs on Mathematical Physics, Cambridge University Press (2015) [arXiv:1404.2601] [INSPIRE].

[55] S.H.S. Alexander, Inflation from D- $\bar{D}$ brane annihilation, Phys. Rev. D 65 (2002) 023507 [hep-th/0105032] [INSPIRE].

[56] L. Randall and R. Sundrum, Out of this world supersymmetry breaking, Nucl. Phys. B 557 (1999) 79 [hep-th/9810155] [INSPIRE].

[57] E. McDonough and M. Scalisi, Inflation from Nilpotent Kähler Corrections, JCAP 11 (2016) 028 [arXiv: 1609.00364] [InSPIRE].

[58] R. Kallosh, A. Linde, D. Roest and Y. Yamada, $\overline{D 3}$ induced geometric inflation, JHEP 07 (2017) 057 [arXiv: 1705. 09247] [INSPIRE].

[59] S. Ferrara, R. Kallosh and A. Linde, Cosmology with Nilpotent Superfields, JHEP 10 (2014) 143 [arXiv: 1408.4096] [INSPIRE].

[60] R. Kallosh and A. Linde, Inflation and Uplifting with Nilpotent Superfields, JCAP 01 (2015) 025 [arXiv: 1408.5950] [INSPIRE].

[61] R.H. Brandenberger, Lectures on the theory of cosmological perturbations, Lect. Notes Phys. 646 (2004) 127 [hep-th/0306071] [INSPIRE].

[62] T. Curtright, Massless Field Supermultiplets With Arbitrary Spin, Phys. Lett. 85B (1979) 219 [INSPIRE]. 
[63] M.A. Vasiliev, 'Gauge' form of description of massless fields with arbitrary spin (in Russian), Yad. Fiz. 32 (1980) 855 [inSPIRE].

[64] S.J. Gates Jr., M.T. Grisaru, M. Roček and W. Siegel, Superspace Or One Thousand and One Lessons in Supersymmetry, Front. Phys. 58 (1983) 1 [hep-th/0108200] [INSPIRE].

[65] I.L. Buchbinder and S.M. Kuzenko, Ideas and methods of supersymmetry and supergravity: Or a walk through superspace, IOP (1998) [INSPIRE].

[66] A. Higuchi, Forbidden Mass Range for Spin-2 Field Theory in de Sitter Space-time, Nucl. Phys. B 282 (1987) 397 [inSPIRE].

[67] S. Deser and A. Waldron, Partial masslessness of higher spins in (A)dS, Nucl. Phys. B 607 (2001) 577 [hep-th/0103198] [INSPIRE].

[68] S.M. Kuzenko, R. Manvelyan and S. Theisen, Off-shell superconformal higher spin multiplets in four dimensions, JHEP 07 (2017) 034 [arXiv: 1701.00682] [INSPIRE].

[69] I.L. Buchbinder, S.J. Gates Jr. and K. Koutrolikos, Higher Spin Superfield interactions with the Chiral Supermultiplet: Conserved Supercurrents and Cubic Vertices, Universe 4 (2018) 6 [arXiv: 1708.06262] [INSPIRE].

[70] J. Hutomo and S.M. Kuzenko, Non-conformal higher spin supercurrents, Phys. Lett. B 778 (2018) 242 [arXiv: 1710.10837] [InSPIRE].

[71] K. Koutrolikos, P. Kočí and R. von Unge, Higher Spin Superfield interactions with Complex linear Supermultiplet: Conserved Supercurrents and Cubic Vertices, JHEP 03 (2018) 119 [arXiv: 1712.05150] [INSPIRE].

[72] I.L. Buchbinder, S.J. Gates Jr. and K. Koutrolikos, Interaction of supersymmetric nonlinear $\sigma$-models with external higher spin superfields via higher spin supercurrents, JHEP 05 (2018) 204 [arXiv: 1804.08539] [inSPIRE].

[73] I.L. Buchbinder, S.J. Gates Jr. and K. Koutrolikos, Conserved higher spin supercurrents for arbitrary spin massless supermultiplets and higher spin superfield cubic interactions, JHEP 08 (2018) 055 [arXiv: 1805. 04413] [INSPIRE].

[74] E.I. Buchbinder, J. Hutomo and S.M. Kuzenko, Higher spin supercurrents in anti-de Sitter space, JHEP 09 (2018) 027 [arXiv: 1805.08055] [INSPIRE].

[75] I.L. Buchbinder, S.J. Gates Jr. and K. Koutrolikos, Integer superspin supercurrents of matter supermultiplets, JHEP 05 (2019) 031 [arXiv: 1811.12858] [INSPIRE].

[76] S.J. Gates and K. Koutrolikos, Progress on cubic interactions of arbitrary superspin supermultiplets via gauge invariant supercurrents, arXiv:1904.13336 [INSPIRE].

[77] J.M. Maldacena, Non-Gaussian features of primordial fluctuations in single field inflationary models, JHEP 05 (2003) 013 [astro-ph/0210603] [INSPIRE].

[78] X. Chen, Primordial Non-Gaussianities from Inflation Models, Adv. Astron. 2010 (2010) 638979 [arXiv: 1002.1416] [INSPIRE].

[79] P. Adshead, R. Easther and E.A. Lim, The 'in-in' Formalism and Cosmological Perturbations, Phys. Rev. D 80 (2009) 083521 [arXiv:0904.4207] [InSPIRE].

[80] Y. Wang, Inflation, Cosmic Perturbations and Non-Gaussianities, Commun. Theor. Phys. 62 (2014) 109 [arXiv: 1303.1523] [INSPIRE].

[81] A. Altland and B.D. Simons, Condensed Matter Field Theory, 2 edition, Cambridge University Press (2010). 
[82] E. Komatsu and D.N. Spergel, Acoustic signatures in the primary microwave background bispectrum, Phys. Rev. D 63 (2001) 063002 [astro-ph/0005036] [InSPIRE].

[83] D. Baumann, G. Goon, H. Lee and G.L. Pimentel, Partially Massless Fields During Inflation, JHEP 04 (2018) 140 [arXiv:1712.06624] [INSPIRE].

[84] CMB-S4 collaboration, CMB-S4 Science Book, First Edition, arXiv:1610.02743 [INSPIRE].

[85] H. Lee, High-Energy Aspects of Inflationary Cosmology, Ph.D. Thesis, Cambridge U., DAMTP (2017) [DOI:10.17863/CAM.20391].

[86] D.H. Lyth and D. Roberts, Cosmological consequences of particle creation during inflation, Phys. Rev. D 57 (1998) 7120 [hep-ph/9609441] [INSPIRE].

[87] N. Bartolo, A. Kehagias, M. Liguori, A. Riotto, M. Shiraishi and V. Tansella, Detecting higher spin fields through statistical anisotropy in the CMB and galaxy power spectra, Phys. Rev. D 97 (2018) 023503 [arXiv: 1709.05695] [INSPIRE].

[88] A. Moradinezhad Dizgah, G. Franciolini, A. Kehagias and A. Riotto, Constraints on long-lived, higher-spin particles from galaxy bispectrum, Phys. Rev. D 98 (2018) 063520 [arXiv: 1805.10247] [INSPIRE].

[89] A. Moradinezhad Dizgah and C. Dvorkin, Scale-Dependent Galaxy Bias from Massive Particles with Spin during Inflation, JCAP 01 (2018) 010 [arXiv:1708.06473] [INSPIRE].

[90] A. Moradinezhad Dizgah, H. Lee, J.B. Muñoz and C. Dvorkin, Galaxy Bispectrum from Massive Spinning Particles, JCAP 05 (2018) 013 [arXiv: 1801.07265] [INSPIRE].

[91] L. Bordin and G. Cabass, Probing higher-spin fields from inflation with higher-order statistics of the CMB, JCAP 06 (2019) 050 [arXiv: 1902.09519] [INSPIRE].

[92] K. Kogai, T. Matsubara, A.J. Nishizawa and Y. Urakawa, Intrinsic galaxy alignment from angular dependent primordial non-Gaussianity, JCAP 08 (2018) 014 [arXiv:1804.06284] [INSPIRE].

[93] A. Kehagias and A. Riotto, On the Inflationary Perturbations of Massive Higher-Spin Fields, JCAP 07 (2017) 046 [arXiv: 1705. 05834] [INSPIRE].

[94] G. Franciolini, A. Kehagias and A. Riotto, Imprints of Spinning Particles on Primordial Cosmological Perturbations, JCAP 02 (2018) 023 [arXiv:1712.06626] [INSPIRE].

[95] G. Franciolini, A. Kehagias, A. Riotto and M. Shiraishi, Detecting higher spin fields through statistical anisotropy in the CMB bispectrum, Phys. Rev. D 98 (2018) 043533 [arXiv: 1803.03814] [INSPIRE].

[96] D. Marolf, L. Martucci and P.J. Silva, Actions and Fermionic symmetries for D-branes in bosonic backgrounds, JHEP 07 (2003) 019 [hep-th/0306066] [INSPIRE].

[97] D. Marolf, L. Martucci and P.J. Silva, Fermions, T duality and effective actions for D-branes in bosonic backgrounds, JHEP 04 (2003) 051 [hep-th/0303209] [INSPIRE].

[98] L. Martucci, J. Rosseel, D. Van den Bleeken and A. Van Proeyen, Dirac actions for D-branes on backgrounds with fluxes, Class. Quant. Grav. 22 (2005) 2745 [hep-th/0504041] [INSPIRE].

[99] K. Dasgupta, M. Emelin and E. McDonough, Fermions on the antibrane: Higher order interactions and spontaneously broken supersymmetry, Phys. Rev. D 95 (2017) 026003 [arXiv: 1601.03409] [INSPIRE]. 
[100] X. Chen, H. Firouzjahi, M.H. Namjoo and M. Sasaki, A Single Field Inflation Model with Large Local Non-Gaussianity, EPL 102 (2013) 59001 [arXiv:1301.5699] [INSPIRE].

[101] R. Holman and A.J. Tolley, Enhanced Non-Gaussianity from Excited Initial States, JCAP 05 (2008) 001 [arXiv: 0710.1302] [INSPIRE].

[102] N. Arkani-Hamed, D. Baumann, H. Lee and G.L. Pimentel, The Cosmological Bootstrap: Inflationary Correlators from Symmetries and Singularities, arXiv:1811.00024 [INSPIRE].

[103] E. Witten, String theory dynamics in various dimensions, Nucl. Phys. B 443 (1995) 85 [hep-th/9503124] [INSPIRE].

[104] P. Hořava and E. Witten, Heterotic and type-I string dynamics from eleven-dimensions, Nucl. Phys. B 460 (1996) 506 [hep-th/9510209] [INSPIRE].

[105] C. Hull and B. Zwiebach, Double Field Theory, JHEP 09 (2009) 099 [arXiv:0904.4664] [INSPIRE].

[106] P.D. Meerburg, J. Meyers, A. van Engelen and Y. Ali-Haïmoud, CMB B-mode non-Gaussianity, Phys. Rev. D 93 (2016) 123511 [arXiv: 1603.02243] [INSPIRE].

[107] S.J. Gates Jr., Sticking with SUSY, Phys. World 27 (2014) 32. 